\title{
Mg abundances in metal-poor halo stars as a tracer of early Galactic mixing ${ }^{\star}$
}

\author{
E. Arnone ${ }^{1,2}$, S. G. Ryan ${ }^{1}$, D. Argast ${ }^{3}$, J. E. Norris ${ }^{4}$, and T. C. Beers ${ }^{5}$ \\ ${ }^{1}$ Dept. of Physics and Astronomy, The Open University, Milton Keynes, MK7 6AA, UK \\ e-mail: s.g.ryan@open.ac.uk \\ 2 Dept. of Physics and Astronomy, University of Leicester, Leicester, LE1 7RH, UK \\ e-mail: e.arnone@ion.le.ac.uk \\ 3 Dept. of Physics and Astronomy, University of Basel, Basel, Switzerland \\ e-mail: argast@astro.unibas.ch \\ ${ }^{4}$ Research School of Astronomy and Astrophysics, The Australian National University, Weston Creek ACT 2611, Australia \\ e-mail: jen@mso.anu.edu.au \\ 5 Dept. of Physics and Astronomy and JINA: Joint Institute for Nuclear Astrophysics, Michigan State University, East Lansing, \\ MI 48824, USA \\ e-mail: beers@pa.msu.edu
}

Received 6 June 2004 / Accepted 27 September 2004

\begin{abstract}
We present results of a detailed chemical analysis performed on 23 main-sequence turnoff stars having $-3.4 \leq$ $[\mathrm{Fe} / \mathrm{H}] \leq-2.2$, a sample selected to be highly homogeneous in $T_{\text {eff }}$ and $\log (g)$. We investigate the efficiency of mixing in the early Galaxy by means of the $[\mathrm{Mg} / \mathrm{Fe}]$ ratio, and find that all values lie within a total range of 0.2 dex, with a standard deviation about the mean of $0.06 \mathrm{dex}$, consistent with measurement errors. This implies there is little or no intrinsic scatter in the early ISM, as suggested also by the most recent results from high-quality VLT observations. These results are in contrast with inhomogeneous Galactic chemical evolution (iGCE) models adopting present supernova (SN) II yields, which predict a peakto-peak scatter in $[\mathrm{Mg} / \mathrm{Fe}]$ as high as 1 dex at very low metallicity, with a corresponding standard deviation of about $0.4 \mathrm{dex}$. We propose that cooling and mixing timescales should be investigated in iGCE models to account for the apparent disagreement with present observations. The contrast between the constancy and small dispersion of $[\mathrm{Mg} / \mathrm{Fe}]$ reported here and the quite different behaviour of $[\mathrm{Ba} / \mathrm{Fe}]$ indicates, according to this interpretation, that $\mathrm{Mg}$ and $\mathrm{Ba}$ are predominantly synthesised in different progenitor mass ranges.
\end{abstract}

Key words. stars: Population II - stars: abundances - Galaxy: formation - Galaxy: evolution - Galaxy: halo

\section{Introduction}

The chemical evolution of the Milky Way arises from the continuous exchange of material between stars and the interstellar medium (ISM). The surfaces of long-lived, low-mass stars retain the composition of the ISM from their formation, so the early phases of the ISM are recorded in metal-poor stars. Metal-poor stars exhibit enhancements of oxygen and other $\alpha$-elements relative to iron, which are explained by the differing yields of supernovae (SN) of type II and type Ia. The O comes from SN II (Thielemann et al. 1990), whereas Fe is produced by both SN Ia and SN II (Nomoto et al. 1984). It is usually inferred that the early Galaxy must have been enriched only by SN II; only more recently have SN Ia started to pollute the ISM, reducing the $\alpha$-enhancement at higher metallicities

$\star$ Table 1 is only available in electronic form at http://www. edpsciences.org
(Tinsley 1980). This implies that the chemical composition of metal-poor halo stars is determined exclusively by SN II events and subsequent ISM mixing in the early Galaxy.

Trends of the abundances of different elements with metallicity are not the only tracers of the enrichment of the Galaxy. The scatter in the relative abundances of low-metallicity stars indicates the level of mixing of the early ISM. Whether all of the observed scatter is an intrinsic property of the ISM, and thus due to incomplete mixing in the early Galaxy, or is possibly due to errors that exceed the formal estimates of uncertainties, is still unclear. Nucleosynthesis models invoke various sources to explain the observed abundances of heavy elements (e.g. Travaglio et al. 2003), rising the level of uncertainty in their nucleosynthesis production. However, for some lighter elements, such as the $\alpha$-elements $\mathrm{O}$ and $\mathrm{Mg}$, their expected modes of synthesis do not predict differences in the ejected yields for stars of similar mass. Any scatter observed in the 
abundances of these elements should thus reflect more directly the effects of enrichment and mixing events in the early Galaxy.

SN II progenitors evolve on timescales of a few to tens of Myrs. In the earliest phase of the Galaxy there may have been a period of star formation during which the zones enriched by SN II have not yet mixed prior to the formation of the next stars. In this case, the newly forming stars will have different chemical compositions. Audouze \& Silk (1995) proposed that, at very low metallicity, clouds in the ISM could have been polluted by a maximum of three SN II, consistent with the suggestion by Ryan et al. (1991b) that single SN were sufficient for the enrichment of the gas that formed stars at $[\mathrm{Fe} / \mathrm{H}] \sim-3.5$. If the early ISM was dominated by local inhomogeneities, as predicted by inhomogeneous Galactic chemical evolution (iGCE) models (e.g. Ishimaru \& Wanajo 1999; Tsujimoto \& Shigeyama 1999; Argast et al. 2000; Travaglio et al. 2001), and the $[\mathrm{O} / \mathrm{Fe}]$ and $[\mathrm{Mg} / \mathrm{Fe}]$ ratios depend on $\mathrm{SN}$-progenitor mass or metallicity, then $[\mathrm{O} / \mathrm{Fe}]$ and $[\mathrm{Mg} / \mathrm{Fe}]$ should show a scatter in the abundances trend versus metallicity, the surface abundances of these stars reflecting the local enrichment of the ISM. Argast finds, for example, that although the ISM is well mixed at $[\mathrm{Fe} / \mathrm{H}]>-2.0$, there is essentially no mixing at $[\mathrm{Fe} / \mathrm{H}]<-3.0$. This gives rise to progressively more star-to-star scatter as $[\mathrm{Fe} / \mathrm{H}]$ decreases below -2.0 .

Although there have been a large number of observational studies, the usually required agglomeration of results from different authors introduces the possibility that the reported scatter of the $\alpha$-element abundance may be due to a lack of internal consistency, as discussed by Norris et al. (2001). Underscoring this possibility, Magain (1987) found that an homogeneous re-analysis of literature data comprising 21 stars over the range $-3.6 \leq[\mathrm{Fe} / \mathrm{H}] \leq-0.9$ gave a mean $\mathrm{Mg}$ abundance $[\mathrm{Mg} / \mathrm{Fe}]=0.45$ and a standard deviation, as a measure of the scatter, of $0.1 \mathrm{dex}$ (scatters of the order $0.15 \mathrm{dex}$ were reported for $[\mathrm{Ca} / \mathrm{Fe}]$ and $[\mathrm{Si} / \mathrm{Fe}])$. Ten of the stars studied by Magain fell in the interval $-3.4 \leq[\mathrm{Fe} / \mathrm{H}] \leq-2.2$ which we study in the present paper. A similar result was found by Nissen et al. (1994) for higher metallicities: $[\mathrm{Mg} / \mathrm{Fe}]=0.41$ and standard deviation $0.07 \mathrm{dex}$, over the range $-2.0 \leq[\mathrm{Fe} / \mathrm{H}] \leq$ -1.0. Recently, Carretta et al. (2002) found that the scatter in their estimate of intermediate mass elemental abundances was compatible with their estimated observational uncertainties. The most recent study of Cayrel et al. (2003), based on extremely high-quality VLT/UVES observations of giant stars from the HK survey, exhibit a low scatter in the abundances of most elements, down to metallicities as low as $[\mathrm{Fe} / \mathrm{H}]=-4.2$. These authors report a standard deviation about their mean $[\mathrm{Mg} / \mathrm{Fe}]$ value of $0.13 \mathrm{dex}$, and as low as $0.05 \mathrm{dex}$ for $[\mathrm{Cr} / \mathrm{Fe}]$. This indeed supports the hypothesis that there is no large scatter in the early ISM.

Other studies, e.g., Fuhrmann et al. (1995) and Mashonkina et al. (2003) have reported higher scatters in the alpha-element ratios. Even though these studies suggest the existence of stars with a different enrichment history (see e.g., Shiegeyama \& Tsujimoto (2003) for a possible origin of metal-poor stars with low $[\alpha / \mathrm{Fe}])$, their average standard deviation is still of the order $\sim 0.1$ dex, far below what is expected in the case of an early inhomogeneous ISM. However, among the eight extremely metal-poor stars $([\mathrm{Fe} / \mathrm{H}]<-3.5)$ considered in the review by Norris (2003), two objects show $[\mathrm{Mg} / \mathrm{Fe}]>1.0$. This indicates the existence of some real differences, but only for metallicity much lower than previously thought.

Clearly, studies of the mixing efficiency in the early Galaxy are fundamental for testing inhomogeneous GCE models. This can be accomplished by a detailed analysis of $[\mathrm{Mg} / \mathrm{Fe}]$ in halo stars, and is the main purpose of this work. We concentrate on $[\mathrm{Mg} / \mathrm{Fe}]$ because of the suitability of $\mathrm{Mg}$ measurements in stars at very low metallicity, and to avoid the still debated uncertainties on measurements of O. Studies of the scatter vs. metallicity for different stellar populations could also allow one to constrain the yields from SN II. Attempts to do this have so far been unsuccessful for individual stars (Chieffi \& Limongi 2002) because of the difficulty of computing SN yields that match the composition of metal-poor stars. François et al. (2004) analyse how SN II yields can be constrained by the observations on the basis of their homogeneous GCE model, suggesting that major revisions of SN II yields are required to match the elemental abundances observed at very low metallicities.

Iron yields are particularly difficult to predict, because Fe production depends on the uncertainties in the mass cuts adopted for SN II models as well as the degree of mixing prior to fallback (Nomoto et al. 1997), whereas Mg is produced in hydrostatic burning much farther out in the star, and hence is subject to fewer uncertainties. As a result, $\mathrm{Mg}$ could also be a better chemical chronometer compared to $\mathrm{Fe}$; its study would aid our understanding of the age-metallicity relation, a key constraint on studies of chemical evolution and nucleosynthesis.

The primary motivation for this study is to produce an independent estimate of the level of elemental scatter in the early Galactic ISM, as unaffected as possible by uncertainties. Unlike the most recent study of Cayrel et al. (2003), whose $S / N$ ratio is higher ( $\geq 200 / 1$, compared to our $100 / 1-150 / 1)$, and who thus obtain better precision on a single measurement, here we focus on the homogeneity of the sample, and demonstrate how our homogeneous chemical analysis leads to a better constraint on the observed scatter of our derived abundances. The aim is to avoid the introduction of star-to-star differences that may lead to increased and misleading errors. We thus concentrate on halo stars that are in the same evolutionary stage, have only small differences in stellar parameters, and make use of the same $\mathrm{Mg}$ absorption lines for all objects. It is of fundamental importance to understand whether the scatter we observe is intrinsic to the sample or due to deficiencies in the analysis. We present the results of the chemical analysis of a sample of main-sequence turnoff halo stars, focusing on $[\mathrm{Fe} / \mathrm{H}]$ and $[\mathrm{Mg} / \mathrm{Fe}]$. We describe the definition of the sample and the observational data in Sect. 2. Particular importance has been given to the use of techniques that produce the highest homogeneity of analysis. The methods adopted and the chemical analysis are presented in Sect. 3. Section 4 reports our results and the determination of their uncertainties. In Sect. 5 we analyse the results and focus on their possible implications. 


\section{Definition of the sample, observations and photometry}

\subsection{Data}

The stellar sample was originally selected by Ryan et al. (1999 - hereafter RNB) from the surveys of Schuster \& Nissen (1988); Ryan (1989); Beers et al. (1992) and Carney et al. (1994), for the purpose of studying Li in metal-poor stars. This sample consists of 23 main-sequence turnoff stars with a narrow range of stellar parameters, the target being effective temperatures $6100 \pm 50 \mathrm{~K} \leq T_{\text {eff }} \leq 6300 \pm 50 \mathrm{~K}$ and metallicities in the range $-3.5 \leq[\mathrm{Fe} / \mathrm{H}] \leq-2.5$. The choice of turnoff stars excludes the presence of subgiants, and reduces the range of logarithmic surface gravity to values within a few tenths of a dex from an expected theoretical $\log (g) \sim 4.0$ dex. Systematic errors introduced in the analysis will thus affect the mean values of our estimates, rather than introducing large star-to-star differences, allowing for high internal consistency in our derived results.

Observations were made with the $3.9 \mathrm{~m}$ Anglo-Australian Telescope (AAT), with the University College London echelle spectrograph (UCLES) - see RNB for details. The stellar spectra are of high resolving power $(R \sim 40000)$, high signal-to-noise ratio $(S / N)$, exceeding $100 / 1$ in most cases, and (incomplete) wavelength coverage over the range $\lambda \approx$ 4900-8200 A. Stars were observed in different epochs in 1996, 1997 and twice in 1998 (hereafter 1998a and b). For 12 of the 23 targets, spectra were co-added from different epochs. At each epoch, a series of multiple observations was made to increase the final $S / N$ obtained.

Photometric measurements are available from a few consistent sources, for Johnson-Cousins UBVRI colours (Ryan 1989), and for Strömgren uvby (Schuster \& Nissen 1988, 1989; Schuster et al. 1993, 1996). For uncertainties and interstellar reddening we adopt the estimates of RNB (and references therein) and calculate the reddening for the $c_{1}$ index as $E\left(c_{1}\right)=$ $0.20 E(b-y)$ (Golay 1974). As discussed below, we use photometric estimates extensively for the stellar parameters due to the very low number of spectral lines in the lowest-metallicity stars. The strengths of the $\mathrm{Mg} b$ lines at 5172 and $5183 \AA$, even at extremely low metallicity, permits $\mathrm{Mg}$ abundance determinations in all of our stars.

\subsection{Galactic populations}

The very low metallicity of most of our program stars is prima facie evidence for their being part of the halo population. However, some recent studies have claimed that the thick disk could extend to include stars of quite low metallicities, $[\mathrm{Fe} / \mathrm{H}] \sim-2.0$, though with very low frequency. See, for example, Allen et al. (1991), Chiba \& Beers (2000), one star of Ibukiyama \& Arimoto (2002), and Beers et al. (2002). Therefore, we further investigate the likely halo membership of our sample stars using the criteria of Feltzing et al. (2003), i.e., adopting a $200 \mathrm{~km} \mathrm{~s}^{-1}$ radius on a Toomre $\left(U W=\left(U^{2}+W^{2}\right)^{1 / 2}\right.$ vs. $V$ ) diagram as the border between the thick disk and halo. This limit is suggested by their data, as well as the data of
Fuhrmann (1998). This is a somewhat more detailed criteria of interpreting the actual three-dimensional motion of the stars, as compared to criteria where only the $V$ component of motion is considered in the identification of the population of the stars (see e.g., Ibukiyama \& Arimoto 2002 for a description of this second method). $U, V$ and $W$ components were computed by Ryan \& Norris (1991a) and by Carney et al. (1994) for the stars in their sample. Heliocentric velocity components from Ryan \& Norris are transformed to the LSR frame by assuming the solar motion relative to the LSR is $(-9,12,7) \mathrm{km} \mathrm{s}^{-1}$ in the directions corresponding to $(U, V, W)$ (Mihalas \& Binney 1981). The analysis in the Toomre diagram shows that all but two of the stars in our sample lie outside the $0-200 \mathrm{~km} \mathrm{~s}^{-1}$ zone, i.e., they are almost certainly halo stars. Of these two exceptions, $\mathrm{BD}+3^{\circ} 740$ has a metallicity of $[\mathrm{Fe} / \mathrm{H}]=-2.78$, surely a halo metallicity, while $\mathrm{BD}+26^{\circ} 3578$ has $[\mathrm{Fe} / \mathrm{H}]=-2.22$, where both estimates are based on high- $S / N 1 \AA$ resolution spectra (RNB). We found that Carney's estimates of $U W$ are lower by 50 to $150 \mathrm{~km} \mathrm{~s}^{-1}$ than those of Ryan and Norris (1991a) for the six stars in common. Fuhrmann et al. (1998) and Ryan et al. (2001) found Carney et al. photometric distances to underestimate the Hipparcos distances in each case for nine stars in common. This possibly explains the difference in the velocity components. A correction was applied to Carney's distances of these two stars, which moved the velocity of BD $+3^{\circ} 740$ into the halo zone, but still left $\mathrm{BD}+26^{\circ} 3578$ in the thick-disk zone. However, our estimate of its metallicity, $[\mathrm{Fe} / \mathrm{H}]=-2.49$, makes it a rather unlikely thick-disk suspect, hence we conclude that we are analysing stars that are sampling exclusively the halo stellar population.

\section{Chemical analysis}

In this section we describe the procedure followed to derive the abundances of chemical elements from the observed spectra, using Kurucz's WIDTH6 code (Kurucz \& Furenlid 1979). The code assumes local thermodynamic equilibrium (LTE) for the formation of the spectral lines. We measure the equivalent width $(E W)$ for each line we consider to be reliable on the basis of a selected list of lines that is described below. A model atmosphere with the appropriate stellar parameters, measured $E W \mathrm{~s}$, and the atomic data for the spectral lines are introduced as inputs to the code. The code then calculates the abundances for each individual line by requiring the calculated $E W$ to match the observed one. The final abundance of a chemical element is the average of the line abundances. We discuss the details of this analysis in the following sub-sections.

\subsection{Line selection and atomic data}

We have given priority to establishing the reliability of each individual spectral line, rather than to achieving a large total number of lines. Our initial selection is based on the lines observed in the solar spectrum (Moore et al. 1966). We reject lines with possible blending. We do not include lines with $g f$-values having reported uncertainties larger than $25 \%$, and discard lines for which, for a large part of the sample, we derive abundances systematically different from the mean abundance of all the lines 
of a specific element. The latter can be due to cases in which the line sits at the very edge of a spectral order, or to poorly known $g f$ data.

Unfortunately, no single source gives laboratory $g f$ values for all lines, so possible systematic differences between atomic data sources could introduce systematic differences in the abundances derived from different lines. For all lines we adopt recent experimental or solar $g f$-values and avoid theoretical ones, because of the large discrepancies that have been found in some cases. Whenever possible, $g f$-values are averaged from several reliable sources. Because of our scientific aims and the paucity of lines of other species, special attention was addressed to Fe I, Fe II and Mg I lines.

Fe I - For Fe I lines we used mainly $g f$-values from O'Brian et al. (1981) and from the Oxford group (see Table 1 for references). However, in attempting to use Fe lines with $g f$-values listed only by Fuhr et al. (1988), we found a systematic difference in the derived abundances of $\sim-0.1 \mathrm{dex}$. We thus do not include lines from Fuhr et al. alone.

$\mathrm{Fe} I I$ - The importance of Fe II lines is to constrain the surface gravity of the star. All but the five most metal-poor stars exhibit Fe II lines in our spectra. We consider Fe II lines at 4923, 5197 and $5276 \AA$, and use Kroll \& Kock (1987) gf values. Unfortunately, only for half of the sample do we obtain good measurements; many Fe II lines are distorted by noise or do not pass the $E W$ threshold described below. Moreover, the Fe II line at $4923 \AA$ was identified only in the observations from 1998a, which have a larger wavelength coverage. A comparison of the abundances derived from this line and from the other two lines do not show any particular difference. Thus, we believe we do not introduce any systematic error in the Fe II abundance for the subset of stars observed in 1998a.

$M g I-$ The Mg Ib triplet around $5172 \AA$ is the most obvious feature in all the stellar spectra of our sample. This allows us to measure Mg lines even in the most metal-poor stars, where typically only four or five lines are usable in the observed spectral region. We thus rely on the triplet to obtain high star-to-star consistency in the derived $\mathrm{Mg}$ abundances. We also compare these with abundances derived from the weak lines at 5528 and $5711 \AA$ whenever we can measure them. For Mg I we adopt the solar $g f$-values of Fuhrmann et al. (1995). These are in excellent agreement with the values of Wiese \& Martin (1980), and the theoretical calculations of Chang (1990) and Chang \& Tang (1990) for the $\mathrm{Mg}$ Ib lines at 5172.698 and $5183.619 \AA$ and for the $\mathrm{Mg}$ I line $5711.092 \AA$. For the line at $5528.42 \AA$, we notice that the $g f$-value of -0.51 used by Fuhrmann et al. is lower than the value -0.341 of Wiese \& Martin, but its adoption leads to higher internal consistency of the $\mathrm{Mg}$ abundances derived from different lines.

The Mg Ib triplet lines are very strong, with extremely broad wings at solar metallicities; even in our low metallicity range the line wings are still moderately strong. Fuhrmann et al. (1995) analyse $\mathrm{Mg}$ I lines at 4571, 4703, 4730 and the triplet at $5172 \mathrm{~mA}$ for 56 metal-poor stars. They report no large systematic differences in the $\mathrm{Mg}$ abundances derived from these strong lines and from weak $\mathrm{Mg}$ lines, nor from lines with different excitation potentials. However, for the three stars where they identify our subset of weak lines (5528 and $5711 \AA$ ), they find abundances derived from the latter to be about $0.05 \mathrm{dex}$ smaller. In our results there is no systematic difference affecting all the stars. Single cases in which we find a difference between weak and strong line $\mathrm{Mg}$ abundances are more likely related to the uncertainties in the model parameters (as discussed below).

The use of the two strong $\mathrm{Mg}$ Ib lines requires a more careful investigation of the damping constant. The classical treatment of the van der Waal's broadening factor by Unsold (1955) has recently been revised. The formalism of Anstee \& O'Mara (1998) leads to $\gamma_{\mathrm{AO}}=2.32 \times \gamma_{\text {Unsold }}$, while the value derived from a fit to the $\mathrm{Mg}$ Ib lines in the spectrum of the Sun is $\gamma_{\odot}=2.04 \times \gamma_{\text {Unsold. }}$. This suggests a $\sim 10 \%$ uncertainty in the damping factor, which corresponds to an uncertainty of the order of 0.02 dex in our $\mathrm{Mg}$ abundances, since our $\mathrm{Mg}$ lines lie at the beginning of the flat part of the curve of growth. We assume an intermediate damping constant of $2.2 \times \gamma_{\text {Unsold }}$. We note that the suggestion of Gratton \& Sneden (1994) of adopting a factor $5 \times \gamma_{\text {Unsold }}$ would yield a worse agreement of the $\mathrm{Mg}$ abundances derived from strong and weak lines in our sample. We do not investigate this possibility any further in this study, and rely instead on the estimates above.

This selection procedure leads to a total of 52 reliable lines (including $4 \mathrm{Mg}, 32 \mathrm{Fe}$ I and $3 \mathrm{Fe}$ II lines), as shown in Table 1. Only eight of these lines (of which three are Mg lines) are measurable in our most metal-deficient stars.

\subsection{Equivalent widths}

We used IRAF to continuum fit the individual orders of the echelle spectra, and to shift the spectra to the rest frame. Avoiding lines distorted by excessive noise or by cosmic rays, the equivalent width $(E W)$ of each line from our list was measured by fitting a Gaussian profile (for lines with $E W \mathrm{~s}$ up to $\sim 100 \AA$ ) and a Voigt profile for stronger lines (i.e., most of the $\mathrm{Mg}$ Ib lines), where the Gaussian profile was inappropriate. In a few cases the adopted $E W$ was the average from Voigt and Gaussian fits. The Voigt fit is much more sensitive than the Gaussian fit to the continuum location. Any systematic error in the continuum fitting would introduce a trend in the $\mathrm{Mg}$ Ib abundances versus line-strength, and thus versus metallicity (the highest-metallicity stars showing the stronger lines). However, for these highest-metallicity stars we always have a weaker $\mathrm{Mg}$ line to check the derived abundances, as discussed below.

To estimate the uncertainties on our measured $E W \mathrm{~s}$, we calculated the standard deviation for each of the 160 pairs of multiple measurements, and obtained a mean standard deviation $\bar{\sigma}=1.6 \mathrm{~m} \AA$. This is in very good agreement with the $2 \mathrm{~mA}$ sensitivity suggested by RNB on the basis of the spectral $S / N$ ratios. For the subset of stronger lines with $E W>70 \mathrm{~m} \AA$, we found a mean standard deviation $\bar{\sigma}_{E W>70 \mathrm{~m}}=2.5 \mathrm{~m} \AA$. The strong Mg Ib lines have $\bar{\sigma}=4 \mathrm{~m} \AA$, corresponding to a $4 \%$ uncertainty in the derived $E W \mathrm{~s}$. 


\subsection{Refining the line measurements}

We filter our line measurements by constraining the line central wavelength, measured equivalent width, and velocity width, $F W H M_{\mathrm{v}}$, corresponding to the Gaussian $F W H M$. We require the centre of the fitted profile to be within $0.05 \AA$ of the central wavelength observed in the solar spectrum (Moore et al. 1966). This allows for an uncertainty in the adopted rest-frame correction, and for a small deviation of the centre of the fit from the core of the line (e.g., as can occur when the fit is dominated by the wings of the profile). We then require a minimum $E W$ of $7.0 \mathrm{~m} \AA$, which is 4.5 times the mean standard deviation obtained for multiple observations, $\bar{\sigma}$. An analysis based on the more conservative criteria of requiring $E W$ larger than $10.0 \mathrm{~m} \AA$ shows an increased scatter in our final abundances; although the higher threshold leads to more precise measurements of individual lines, the number of accepted lines decreases, in particular for the most metal-poor stars. The lines were also filtered by requiring $7.0 \leq F W H M_{\mathrm{v}} \leq 14.0 \mathrm{~km} \mathrm{~s}^{-1}$ in order to avoid blends, or lines so distorted by noise to appear either narrower than the instrumental profile or unphysically wide. This selection should lead to higher internal consistency of the measurements. The final $E W \mathrm{~s}$ are reported in Table 1. For each line we list the chemical species (el), central wavelength $(\lambda)$, adopted $g f$-value and corresponding reference (ref), and the equivalent width measured in each program star.

\subsection{Model atmosphere}

We adopt the grid of 1D, LTE model atmospheres of Kurucz (1989, private communication), interpolating within the original models to our required stellar parameters. As previously noted (Ryan et al. 1996), abundances derived with Kurucz's 1989 models are in good agreement with abundances derived with the model atmospheres of Bell et al. (1981), but newer Kurucz (1993) models have a different temperature structure and lead to abundances higher than the previous models. Ryan et al. (1996) found that temperatures are up to $200 \mathrm{~K}$ higher in the line-forming region of the Kurucz (1993) models and result in +0.1 dex in their Fe I abundances for turn-off stars. We find a +0.1 dex difference for lines of $E W \sim 10 \mathrm{~m} \AA$, rising to $+0.15 \mathrm{dex}$ at $\sim 40 \mathrm{~m} \AA$ and to +0.2 dex at $\sim 150 \mathrm{~m} \AA$, the wings of stronger lines being more sensitive to these regions. The two strong $\mathrm{Mg}$ Ib lines are thus more sensitive to the change in the temperature structure of the model than the bulk of Fe lines. This would imply a typical -0.05 dex difference in the $[\mathrm{Mg} / \mathrm{Fe}]$ ratio if we derived the abundances with the Kurucz (1993) models and only the b lines of Mg I. However, the use of weak $\mathrm{Mg}$ lines allows us also to use estimates for the abundance from lines in the same line-forming region as the $\mathrm{Fe}$ lines, and thus obtain a $[\mathrm{Mg} / \mathrm{Fe}]$ value that is less dependent on the temperature structure of the model. The average of $\mathrm{Mg}$ abundances from weak and strong lines reduces the dependence of our results on the adopted model. Moreover, for the lowest-metallicity stars (where weak $\mathrm{Mg}$ lines are not measurable), the $\mathrm{Mg} \mathrm{Ib}$ lines are also weaker and thus less dependent on the temperature structure. We estimate that if we were to adopt Kurucz (1993) models, the standard deviation about the

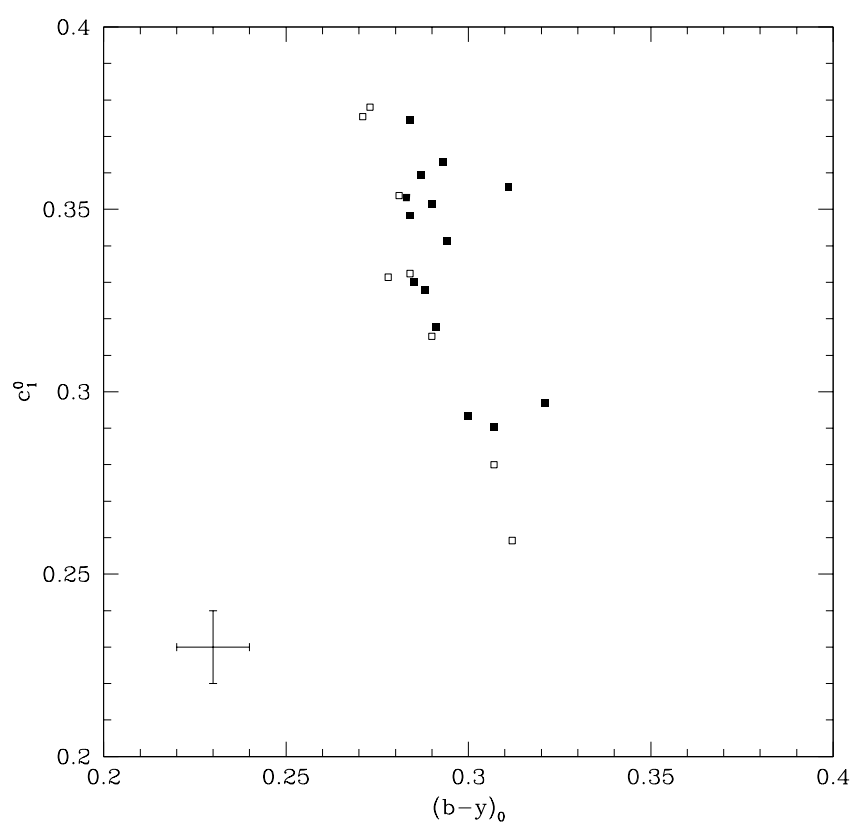

Fig. 1. The position in the diagram dereddened $\mathrm{c}_{1}$ vs. $b-y$ is shown as proof of the similar evolutionary stage of the stars. Filled squares represent stars with measurements of Fe II lines and thus spectroscopic estimate of $\log (g)$. Empty squares are used for stars with no Fe II measurements.

mean $[\mathrm{Mg} / \mathrm{Fe}]$ would increase by less than 0.005 dex. We can thus safely neglect the model dependence in our discussion of the observed scatter.

\subsection{Stellar parameters}

In principle, we can derive the effective temperature, $T_{\text {eff }}$, in an iterative fashion by computing abundances for several models, and requiring no dependence of the Fe abundance on excitation potential (EP). The surface gravity $\log (g)$ can be set by requiring the $\mathrm{Fe}$ abundance derived from $\mathrm{Fe}$ II lines to match that from Fe I lines. Finally, the microturbulent velocity can be derived by requiring no trend of the abundances versus $E W$. However, these spectroscopic estimates for the stellar parameters rely on the measurement of at least some lines of both Fe I and Fe II. The higher the number of lines, the higher the precision of the estimate. As discussed before, the very metal-poor stars show just four or five reliable lines in the stellar spectra; for about half of the sample we cannot measure any Fe II lines at all. Hence, we are not able to derive spectroscopic estimates of the parameters for all the stars and furthermore, when we do have estimates, not all have the same precision.

Photometry can assist us in the task of determining the stellar parameters, having the excellent characteristic of not biasing our precision at lowest metallicity (as is the case for spectroscopic estimates, which rely on a decreasing number of lines at lowest metallicity). As shown in Fig. 1, the stars are in a region of the $c_{1}$ vs. $b-y$ diagram that led RNB to identify them as main-sequence turnoff stars. This determination is done by comparing the observations with evolutionary tracks, on the basis of the $b-y$ temperature sensitive index and the gravity sensitivity of the $c_{1}$ index. We go a step further, and obtain estimates 
for the surface gravity by calibrating the $c_{1}$ index with stars whose spectroscopic gravities we are able to derive. Because of the small number of Fe I lines and the absence of Fe II lines for the lowest-metallicity stars, we adopt photometric calibrations both for $T_{\text {eff }}$ and $\log (g)$, based on the homogeneity of the sample, rather than using solely spectroscopic values. We discuss the details of our method below.

\section{Effective temperature}

The effective temperature is adopted from RNB. Their photometric estimates were based on six indices $(B-V, V-R$, $R-I, \beta, \mathrm{HP} 2$ and $b-y$ ), calibrated with the theoretical calculations of Bell \& Oke (1986) and the empirical relations of Magain (1987). The photometric indices were de-reddened using the reddening maps of Lucke (1978) and Burstein \& Heiles (1982), with Johnson photometric distances from Carney et al. (1994) and Ryan et al. (1989), and the comparison of $b-y$ with the reddening-free index $\beta$ for Strömgren indices. A correction was applied to account for a 0.020 mag difference between the colour excess estimate derived from Strömgren indices and that inferred from reddening maps, where we expect $E(b-y)=$ $0.7 E(B-V)$. The uncertainties on the photometric indices were assumed to be on average less than \pm 0.01 magnitudes (see RNB and references therein for details). The final $T_{\text {eff }}$ estimates are shown in Table 2 . They exhibit a very low relative uncertainty, on the of order $\pm 40 \mathrm{~K}$, although larger systematic errors could affect the zero point by up to $\sim 100$ to $200 \mathrm{~K}$.

Later spectroscopic reanalysis of some stars in our sample have suggested higher temperatures (see e.g., Nissen et al. 2001; Ford et al. 2002). We note the problem of systematic differences between photometric and spectroscopic estimates of $T_{\text {eff }}$, the former being typically expected to be $100 \mathrm{~K}$ hotter according to Alonso et al. (1999). Johnson (2002) finds up to $150 \mathrm{~K}$ difference between $T_{\text {eff }}$ estimates derived with spectroscopy or with photometry. We therefore determine a spectroscopic estimate of the $T_{\text {eff }}$ for the stars by requiring no dependence of the abundance derived from Fe I lines versus the excitation potential (EP) of the line. For most of the stars, there are too few Fe I lines to have a high confidence in the determination of spectroscopic $T_{\text {spec }}$; we work on the subset of 15 stars with more than 10 lines. Our estimates for the effective temperature $T_{\text {spec }}$ are on average $80 \mathrm{~K}$ lower than the temperatures derived by RNB. This is just at the limit of our average $1 \sigma$ statistical error of $80 \mathrm{~K}$ in the derivation of $T_{\text {spec }}$. However, in most cases we have only two or three Fe I lines with high EP, hence the procedure is heavily dependent on these few lines. We thus rely instead on the photometric estimates from RNB, and assess the impact of a possible systematic error up to $150 \mathrm{~K}$, as suggested by the largest differences between RNB estimates and our spectroscopic determinations. We assume a $40 \mathrm{~K}$ random error on the temperature from RNB.

\section{Surface gravity and microturbulent velocity}

We adopt an iterative procedure to determine both the surface gravity and the microturbulent velocity of each star. We make calculations with $\log (g)=4.0$ and $\xi=1.0-1.5 \mathrm{~km} \mathrm{~s}^{-1}$ as our first stage. With these results we calculate the best
Table 2. Model parameters.

\begin{tabular}{|c|c|c|c|c|c|}
\hline Star & $\bar{T} T_{\mathrm{RNB}}$ & $\overline{\sigma_{T}}$ & $\begin{array}{c}\xi \xi \\
( \pm 0.2)\end{array}$ & $\overline{\log (g)_{\text {spec }}}$ & $\begin{array}{c}\log (g)_{\text {phot }} \\
( \pm 0.2)\end{array}$ \\
\hline BD-133442 & 6210 & 30 & 1.5 & - & 3.6 \\
\hline $\mathrm{BD}+1^{\circ} 2341 \mathrm{p}$ & 6260 & 40 & 1.5 & 3.8 & 3.8 \\
\hline $\mathrm{BD}+20^{\circ} 2030$ & 6200 & 40 & 1.6 & 3.9 & 3.9 \\
\hline $\mathrm{BD}+24^{\circ} 1676$ & 6170 & 30 & 1.8 & 3.7 & 3.7 \\
\hline $\mathrm{BD}+26^{\circ} 2621$ & 6150 & 40 & 1.6 & - & 4.0 \\
\hline $\mathrm{BD}+26^{\circ} 3578$ & 6150 & 40 & 1.5 & 3.4 & 3.7 \\
\hline $\mathrm{BD}+3^{\circ} 740$ & 6240 & 40 & 1.8 & 3.7 & 3.7 \\
\hline $\mathrm{BD}+9^{\circ} 2190$ & 6250 & 30 & 1.3 & 3.8 & 3.6 \\
\hline 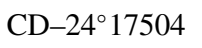 & 6070 & 30 & 1.8 & - & 4.2 \\
\hline $\mathrm{CD}-33^{\circ} 1173$ & 6250 & 20 & 1.8 & - & 3.7 \\
\hline $\mathrm{CD}-35^{\circ} 14849$ & 6060 & 20 & 1.3 & 4.0 & 4.1 \\
\hline $\mathrm{CD}-71^{\circ} 1234$ & 6190 & 30 & 1.5 & 4.1 & 4.1 \\
\hline CS22943-045 & 6140 & 40 & 1.5 & 3.7 & 3.9 \\
\hline G126-52 & 6210 & 40 & 1.8 & 3.9 & 3.8 \\
\hline G4-37 & 6050 & 40 & 1.8 & - & 4.1 \\
\hline G64-12 & 6220 & 30 & 1.7 & - & 3.9 \\
\hline G64-37 & 6240 & 30 & 1.0 & 3.9 & 3.9 \\
\hline HD74000 & 6040 & 30 & 1.3 & 4.2 & 4.1 \\
\hline HD84937 & 6160 & 30 & 1.8 & 3.9 & 3.7 \\
\hline LP635-14 & 6270 & 30 & 1.7 & 4.4 & 3.7 \\
\hline LP651-4 & 6240 & 30 & 1.0 & - & 3.9 \\
\hline LP815-43 & 6340 & 30 & 1.0 & - & 3.6 \\
\hline LP831-70 & 6050 & 20 & 1.0 & - & 4.3 \\
\hline
\end{tabular}

estimate for $\xi$ by requiring no trend of the abundances of Fe I with the $E W$ of the line the abundance was derived from. Due to the lack of lines for the most metal-poor stars, we adopt a range for $\xi$ of $1.0-1.5 \mathrm{~s}^{-1}$ to compensate for the poor spectroscopic determination. We perform calculations with the assumed $\log (g) \pm 0.2$ dex and the calculated $\xi$, searching for the value of $\log (g)$ that would let the $\mathrm{Fe}$ abundances derived from Fe I match the ones from Fe II lines. We then iterate this procedure to obtain new estimates of $\xi$ and $\log (g)$, finding a convergence of the results after a few iterations.

Only one or two Fe II lines are available for each $\log (g)$ determination. The standard error in the difference $[\mathrm{Fe} / \mathrm{H}]_{\mathrm{I}}-[\mathrm{Fe} / \mathrm{H}]_{\mathrm{II}}$ corresponds to an error $\sigma_{\log (g)_{\mathrm{Fe}}} \sim$ 0.12 dex. The inferred $\log (g)$ value is also affected by the adopted effective temperature. Changes in $T_{\text {eff }}$ of $40 \mathrm{~K}$ cause a variation in the inferred $\log (g)$ of $\sigma_{\log (g)_{T}} \sim 0.02$ dex. Values generally adopted for $\xi$ in the case of turn-off stars are in the range $1.0-1.5 \mathrm{~km} \mathrm{~s}^{-1}$. A conservative assumption of a variation of $\xi$, over the range $\Delta \xi= \pm 0.2 \mathrm{~km} \mathrm{~s}^{-1}$, produces a variation in the derived $\log (g)$ of \pm 0.06 dex on average. On the other hand, a variation of $\log (g)$ of \pm 0.06 produces a variation in $\xi$ of less than $0.03 \mathrm{~km} \mathrm{~s}^{-1}$. Thus the determination of $\xi$ is quite insensitive to the adopted $\log (g)$. We expect this 0.06 dex uncertainty on $\log (g)$ to arise only in the worse cases when we cannot constrain $\xi$; in most cases this iterative procedure produces an uncertainty in the derived $\log (g)$ of $\sigma_{\log (g)_{\text {procedure }}} \sim 0.04$ dex. We thus assume a random error on the spectroscopic $\log (g)$ of $\sigma_{\log (g)_{\text {spec }}}=\sqrt{\sigma_{\log (g)_{\mathrm{Fe}}}^{2}+\sigma_{\log (g)_{T}}^{2}+\sigma_{\log (g)_{\text {procedure }}}^{2}}=0.13$. For $\xi$ we adopt the more conservative uncertainty of $0.2 \mathrm{~km} \mathrm{~s}^{-1}$. 


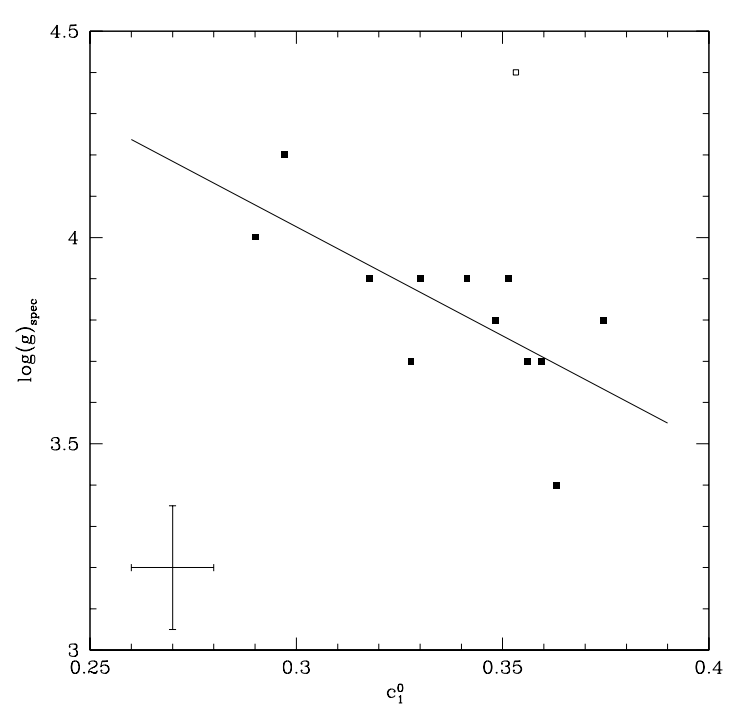

Fig. 2. Calibration of the spectroscopic $\log (g)$ vs. the gravity sensitive dereddened $c_{1}$ photometric index. The empty square represents the star LP635-14 that was rejected from the fit by a $2 \sigma$ clipping procedure.

These spectroscopic estimates of $\log (g)$ are used to calibrate $\log (g)$ versus the de-reddened $c_{1}$ index using a linear fit (see Fig. 2). The $c_{1}$ index was de-reddened adopting the colour excess law $E\left(c_{1}\right)=0.20 E(B-V)$ and $c_{1,0}=c_{1}-E\left(c_{1}\right)$ (Golay 1974). The calibration is then used to calculate "photometric" estimates of the $\log (g)$ for each star (Table 2). The standard deviation about the fit for the calibration of $\log (g)$ versus $c_{1}$ is $0.15 \mathrm{dex}$, excluding (by a $2 \sigma$ clipping procedure) the $\log (g)=4.4$ derived spectroscopically from just one Fe II line for LP635-14. The error on the photometric estimates of $\log (g)$ is adopted to be $0.2 \mathrm{dex}$, as the quadratic sum of the uncertainty on the spectroscopic $\log (g)(0.13)$ and of the calibration versus $c_{1}(0.15)$, the influence of the uncertainty on the $c_{1}$ value being negligible. We then derive the abundances with the adopted photometric $\log (g)$ for the entire sample of stars. For the subset of stars for which we could calculate the spectroscopic $\log (g)$, we perform a further analysis with that value of $\log (g)$, but find no significant difference in the overall trend or scatter of the results.

We investigate a possible dependence of the $c_{1}$ index on temperature as well as gravity, but find no evidence of a correlation between the residuals of the gravity calibration and the temperature scale. This indicates that the gravity sensitivity of the $c_{1}$ index is far more important than its temperature sensitivity over the narrow temperature range of our stars, and the latter does not affect our calibration.

\section{Initial chemical composition}

We also explored the possible sensitivity of the derived abundances on the initial chemical composition adopted for the calculations. The chemical composition is introduced in the model atmosphere by scaling the solar composition with the metallicity of the star. However, because of the very low metallicity of our stars, no significant change in the derived abundances is produced by adopting models with slightly different chemical composition.

\section{Chemical abundances}

We now derive surface abundances from lines of $\mathrm{Mg}$ I and Fe I for our entire sample of stars. For most of the stars we are also able to derive surface abundances for the elements $\mathrm{Ca}$, $\mathrm{Ti}$, and $\mathrm{Cr}$, depending on the presence of reliable spectral lines in our wavelength coverage. The results are listed in Tables 3 and 4 , along with the number of lines used, as well as the standard deviation, $\sigma$, of the abundances derived from different lines. The total uncertainty of each element is described below.

\subsection{Dependence of abundances on model atmosphere parameters}

The dependence of our derived abundances on stellar parameters varies with the strength of the lines used. Because of the homogeneity of our sample, we study this dependence for the whole sample rather than for single stars.

For Fe I, the number of weak lines is far higher than the number of stronger ones. This reduces the sensitivity to strong lines, hence there will be no large differences of sensitivity between higher and lower metallicity stars. Fe II lines are never stronger than $60 \mathrm{m \AA}$, so again the averaged Fe II ensitivity is a good representative for all the stars. We derive abundances for a grid of models in which we vary independently one stellar parameter at a time by its standard error, as discussed in Sect. 3.5, and report the average results for Fe lines in Table 5.

In the case of $\mathrm{Mg}$, the model parameter sensitivity is much more significant in the $\mathrm{Mg} \mathrm{Ib}$ lines than in the weak $\mathrm{Mg}$ line. Besides a dependence on temperature similar to that of Fe I lines, $\mathrm{Mg}$ Ib lines show an extremely high sensitivity to surface gravity because of the stronger wings. We show in Table 5 the average results for the weak Mg I line at $5528 \AA$ and for the average $\mathrm{Mg}$ Ib lines. The dependence of the $[\mathrm{Mg} / \mathrm{Fe}] \mathrm{ra}-$ tio is averaged on the whole sample of stars, and thus weighted on both the weak line and the two strong lines.

Estimating uncertainties by varying just one parameter at a time is a useful exercise, but it does not reflect the real analysis procedure. In order to estimate more realistic uncertainties, we performed a Monte Carlo simulation reproducing the procedure adopted for deriving $\log (g)$ and $\xi$. We consider two candidate stars, $\mathrm{BD}+3^{\circ} 740(10 \mathrm{Fe} \mathrm{I}$ lines, $[\mathrm{Fe} / \mathrm{H}]=-2.84)$ and $\mathrm{BD}+26^{\circ} 3578(21 \mathrm{Fe} \mathrm{I}$ lines, $[\mathrm{Fe} / \mathrm{H}]=-2.49)$ as representative of our sample. For each star we create a set of 25 virtual "observations" by adding numerical noise to the measured $E W$ s. For each line, the distribution of the 25 randomly generated errors added to the $E W$ is a Gaussian with $\sigma=1.5 \mathrm{~m} \AA$ (i.e., our average inferred $1 \sigma$ uncertainty on the $E W$ ). By deriving the surface gravity and microturbulent velocity for the virtual observations with the iterative method used for the real data, we were able to estimate the dependence of our abundances and derived parameters on observational errors, by means of the standard deviation of the results for the 25 simulations. In Table 6 we report the results of this "end-to-end" test of our procedures.

From this test we find that the sensitivity of our results is lower than a quadratic sum of the single parameter dependences estimated one at a time. This is due to the fact that 
Table 3. Fe and Mg chemical abundances.

\begin{tabular}{l|cccccccccc}
\hline \hline Star & {$[\mathrm{Fe} / \mathrm{H}]_{\mathrm{I}}$} & $n_{\mathrm{FeI}}$ & $\sigma_{\text {FeI }}$ & {$[\mathrm{Fe} / \mathrm{H}]_{\mathrm{II}}$} & $n_{\text {FeII }}$ & $\sigma_{\text {FeII }}$ & {$[\mathrm{Mg} / \mathrm{H}]$} & $n_{\mathrm{Mg}}$ & $\sigma_{\mathrm{Mg}}$ & {$[\mathrm{Mg} / \mathrm{Fe}]$} \\
\hline $\mathrm{BD}-13^{\circ} 3442$ & -2.83 & 9 & 0.02 & - & 0 & - & -2.47 & 3 & 0.03 & 0.36 \\
$\mathrm{BD}+1^{\circ} 2341 \mathrm{p}$ & -2.88 & 11 & 0.02 & -2.85 & 1 & - & -2.51 & 3 & 0.01 & 0.37 \\
$\mathrm{BD}+20^{\circ} 2030$ & -2.68 & 16 & 0.01 & -2.68 & 1 & - & -2.39 & 3 & 0.06 & 0.29 \\
$\mathrm{BD}+24^{\circ} 1676$ & -2.60 & 15 & 0.02 & -2.60 & 1 & - & -2.28 & 3 & 0.01 & 0.32 \\
$\mathrm{BD}+26^{\circ} 2621$ & -2.86 & 7 & 0.01 & - & 0 & - & -2.59 & 3 & 0.01 & 0.27 \\
$\mathrm{BD}+26^{\circ} 3578$ & -2.49 & 21 & 0.01 & -2.40 & 1 & - & -2.16 & 2 & 0.01 & 0.33 \\
$\mathrm{BD}+3^{\circ} 740$ & -2.84 & 10 & 0.01 & -2.87 & 2 & 0.09 & -2.52 & 3 & 0.03 & 0.32 \\
$\mathrm{BD}+9^{\circ} 2190$ & -2.83 & 10 & 0.02 & -2.87 & 2 & 0.07 & -2.61 & 3 & 0.07 & 0.22 \\
$\mathrm{CD}-24^{\circ} 17504$ & -3.45 & 5 & 0.03 & - & 0 & - & -3.17 & 3 & 0.10 & 0.28 \\
$\mathrm{CD}-33^{\circ} 1173$ & -3.10 & 8 & 0.03 & - & 0 & - & -2.81 & 3 & 0.04 & 0.29 \\
$\mathrm{CD}-35^{\circ} 14849$ & -2.55 & 20 & 0.01 & -2.53 & 2 & - & -2.31 & 2 & 0.01 & 0.24 \\
$\mathrm{CD}-71^{\circ} 1234$ & -2.55 & 22 & 0.01 & -2.56 & 3 & 0.01 & -2.35 & 3 & 0.03 & 0.20 \\
$\mathrm{CS} 22943-045$ & -2.50 & 22 & 0.02 & -2.43 & 2 & 0.08 & -2.13 & 3 & 0.02 & 0.37 \\
$\mathrm{G} 126-52$ & -2.44 & 20 & 0.01 & -2.49 & 1 & - & -2.17 & 2 & 0.05 & 0.27 \\
$\mathrm{G} 4-37$ & -2.75 & 12 & 0.02 & - & 0 & - & -2.48 & 2 & 0.00 & 0.27 \\
$\mathrm{G} 64-12$ & -3.35 & 4 & 0.04 & - & 0 & - & -3.08 & 3 & 0.05 & 0.27 \\
$\mathrm{G} 64-37$ & -3.15 & 8 & 0.02 & -3.13 & 1 & - & -2.92 & 3 & 0.04 & 0.23 \\
$\mathrm{HD} 74000$ & -2.17 & 28 & 0.01 & -2.24 & 1 & - & -1.95 & 3 & 0.07 & 0.22 \\
$\mathrm{HD} 84937$ & -2.36 & 23 & 0.01 & -2.44 & 3 & 0.01 & -2.01 & 3 & 0.02 & 0.35 \\
LP635-14 & -2.53 & 23 & 0.02 & -2.80 & 1 & - & -2.19 & 3 & 0.02 & 0.34 \\
LP651-4 & -2.69 & 14 & 0.03 & - & 0 & 0.04 & -2.27 & 3 & 0.04 & 0.42 \\
LP815-43 & -2.81 & 7 & 0.01 & - & 0 & - & -2.42 & 2 & 0.09 & 0.39 \\
LP831-70 & -3.06 & 9 & 0.03 & - & 0 & - & -2.69 & 3 & 0.06 & 0.37 \\
\hline
\end{tabular}

${ }^{1}$ In HD74000 we measured the $\mathrm{Mg}$ weak line at $5711 \mathrm{~m} \AA$ instead of the line at $5528 \mathrm{~m} \AA$.

Table 4. $\mathrm{Ca}$, Ti and $\mathrm{Cr}$ chemical abundances. Ti II abundances are derived only in 3 stars.

\begin{tabular}{l|ccccccccc}
\hline \hline Star & {$[\mathrm{Ca} / \mathrm{H}]$} & $n_{\mathrm{Ca}}$ & $\sigma_{\mathrm{Ca}}$ & {$[\mathrm{Ti} / \mathrm{H}]$} & $n_{\mathrm{Ti}}$ & $\sigma_{\mathrm{Ti}}$ & {$[\mathrm{Cr} / \mathrm{H}]$} & $n_{\mathrm{Cr}}$ & $\sigma_{\mathrm{Cr}}$ \\
\hline $\mathrm{BD}-13^{\circ} 3442$ & -2.4 & 3 & 0.03 & - & 0 & - & - & 0 & - \\
$\mathrm{BD}+1^{\circ} 2341 \mathrm{p}$ & -2.37 & 3 & 0.04 & -2.25 & 1 & - & -2.84 & 2 & 0.01 \\
$\mathrm{BD}+20^{\circ} 2030$ & -2.23 & 3 & 0.04 & -2.16 & 1 & - & -2.62 & 2 & 0.04 \\
$\mathrm{BD}+24^{\circ} 1676$ & -2.21 & 3 & 0.03 & $-2.07^{1}$ & 1 & - & -2.72 & 2 & 0.08 \\
$\mathrm{BD}+26^{\circ} 2621$ & -2.67 & 1 & - & - & 0 & - & - & 0 & - \\
$\mathrm{BD}+26^{\circ} 3578$ & -2.05 & 2 & 0.02 & -2.10 & 1 & - & -2.62 & 2 & 0.04 \\
$\mathrm{BD}+3^{\circ} 740$ & -2.43 & 2 & 0.01 & - & 0 & - & - & 0 & - \\
$\mathrm{BD}+9^{\circ} 2190$ & -2.51 & 3 & 0.06 & - & 0 & - & - & 0 & - \\
$\mathrm{CD}-24^{\circ} 17504$ & - & 0 & - & - & 0 & - & - & 0 & - \\
$\mathrm{CD}-33^{\circ} 1173$ & -2.69 & 1 & - & - & 0 & - & -2.96 & 1 & 0.0 \\
$\mathrm{CD}-35^{\circ} 14849$ & -2.22 & 1 & - & - & 0 & - & -2.69 & 2 & 0.07 \\
$\mathrm{CD}-71^{\circ} 1234$ & -2.23 & 3 & 0.02 & - & 0 & - & -2.51 & 3 & 0.18 \\
$\mathrm{CS} 22943-045$ & -1.99 & 5 & 0.05 & $-2.08^{2}$ & 1 & - & -2.57 & 2 & 0.04 \\
$\mathrm{G} 126-52$ & -2.09 & 2 & 0.00 & -2.07 & 1 & - & -2.54 & 1 & - \\
$\mathrm{G} 4-37$ & -2.39 & 1 & - & -2.36 & 1 & - & -2.81 & 2 & 0.04 \\
$\mathrm{G} 64-12$ & -2.87 & 1 & - & - & 0 & - & - & 0 & - \\
$\mathrm{G} 64-37$ & -2.86 & 1 & - & - & 0 & - & - & 0 & - \\
$\mathrm{HD} 74000$ & -1.93 & 4 & 0.05 & -1.91 & 2 & 0.03 & -2.24 & 3 & 0.08 \\
$\mathrm{HD} 84937$ & -1.94 & 4 & 0.04 & $-1.97^{3}$ & 1 & - & -2.44 & 2 & 0.04 \\
$\mathrm{LP} 635-14$ & -2.22 & 3 & 0.02 & -1.78 & 1 & - & -2.63 & 2 & 0.04 \\
$\mathrm{LP} 651-4$ & -2.43 & 2 & 0.07 & - & 0 & - & - & 0 & - \\
$\mathrm{LP} 815-43$ & -2.47 & 1 & - & - & 0 & - & - & 0 & - \\
$\mathrm{LP} 831-70$ & - & 0 & - & -2.46 & 1 & - & -3.20 & 1 & - \\
\hline
\end{tabular}

${ }^{1}[\mathrm{Ti} / \mathrm{H}]_{\mathrm{II}}=-2.19,{ }^{2}:[\mathrm{Ti} / \mathrm{H}]_{\mathrm{II}}=-2.09$ and $^{3}:[\mathrm{Ti} / \mathrm{H}]_{\mathrm{II}}=-2.03$. 
Table 5. Dependence of abundances on model parameters.

\begin{tabular}{lcccccc}
\hline \hline Parameter & Variation & $\overline{\Delta[\mathrm{Fe} / \mathrm{H}]_{\mathrm{I}}}$ & $\overline{\Delta[\mathrm{Fe} / \mathrm{H}]_{\mathrm{II}}}$ & $\overline{\Delta[\mathrm{Mg} / \mathrm{H}]_{5528}}$ & $\overline{\Delta[\mathrm{Mg} / \mathrm{H}]_{\mathrm{Ib}}}$ & $\overline{\Delta[\mathrm{Mg} / \mathrm{Fe}]}$ \\
\hline$T_{\text {eff }}$ (random) & $\pm 40 \mathrm{~K}$ & \pm 0.03 & \pm 0.00 & \pm 0.02 & \pm 0.04 & \pm 0.00 \\
$T_{\text {eff }}$ (systematic) & $\pm 150 \mathrm{~K}$ & \pm 0.13 & \pm 0.02 & \pm 0.07 & \pm 0.14 & \pm 0.01 \\
$\xi$ & \pm 0.2 & $\mp 0.01$ & \pm 0.00 & $\mp 0.01$ & $\mp 0.03$ & $\mp 0.02$ \\
$\log (g)$ & \pm 0.2 & $\mp 0.01$ & \pm 0.03 & $\mp 0.02$ & $\mp 0.10$ & $\mp 0.06$ \\
\hline
\end{tabular}

Table 6. Standard deviations for the results of the Monte Carlo test.

\begin{tabular}{lccccccccc}
\hline \hline Star & {$[\mathrm{Fe} / \mathrm{H}]$} & $\sigma_{E W} / \mathrm{m} \AA$ & $\sigma_{\xi}$ & $\sigma_{\log }(g)$ & $\sigma_{[\mathrm{Fe} / \mathrm{H}]_{\mathrm{I}}}$ & $\sigma_{[\mathrm{Fe} / \mathrm{H}]_{\mathrm{II}}}$ & $\sigma_{[\mathrm{Mg} / \mathrm{H}]}$ & $\sigma_{[\mathrm{Mg} / \mathrm{Fe}]}$ & $\sigma_{[\mathrm{Ca} / \mathrm{Fe}]}$ \\
\hline $\mathrm{BD}+3^{\circ} 740$ & -2.84 & 1.5 & 0.20 & 0.19 & 0.02 & 0.03 & 0.06 & 0.06 & 0.02 \\
$\mathrm{BD}+26^{\circ} 3578$ & -2.49 & 1.5 & 0.17 & 0.19 & 0.02 & 0.03 & 0.04 & 0.03 & 0.02 \\
\hline
\end{tabular}

the errors in the parameters derived with the iterative procedure are not independent, so the parameters partially compensate for one another. Moreover, the abundances are averaged on both weak and stronger lines, decreasing the sensitivity that strong lines would show alone. It is comforting to notice that the standard deviation of the $\xi$ and $\log (g)$ values are in the order of $0.2 \mathrm{~km} \mathrm{~s}^{-1}$ and 0.2 dex respectively, as expected from the procedure used in Sect. 3.5. More interestingly, the resulting dependence of the abundances on the $E W$ uncertainties and, in turn, on these two imperfectly determined parameters, is very small, of the order $0.02 \mathrm{dex}$ for $[\mathrm{Fe} / \mathrm{H}]$ and $0.04-0.06 \mathrm{dex}$ for $[\mathrm{Mg} / \mathrm{H}]$. The real impact of the parameter determination is thus much smaller than Table 5 suggested. This procedure is also useful for evaluating how these errors cancel out in the ratio of the two elements, since we are interested in determining the $[\mathrm{Mg} / \mathrm{Fe}]$ ratio; the uncertainty varies between $0.03 \mathrm{dex}$ and 0.06 dex for stars of higher and lower metallicity. We reemphasize that we did not derive $T_{\text {eff }}$ spectroscopically in this process, because of the lack of lines of high EP. Consequently, an uncertainty from the adopted $T_{\text {eff }}$ must still be included, which we take from Table 5. However, this uncertainty almost cancels out for the $[\mathrm{Mg} / \mathrm{Fe}]$ ratio.

\subsection{Non-LTE effects on the abundances}

We derive chemical abundances assuming local thermodynamical equilibrium (LTE). This assumption may be unrealistic, and one should be careful that lines may form in conditions that depart from LTE. However, the current efforts to remove the LTE assumption yield conflicting results, as shown by the results of Thévenin \& Idiart (1999) and Gratton et al. (1999) for Fe, and Zhao et al. (1998) for Mg I.

Non-LTE (NLTE) calculations from Thévenin \& Idiart (1999) show that iron abundances derived from Fe I lines should be increased by 0.3 dex, compared to the LTE estimates, for $[\mathrm{Fe} / \mathrm{H}]$ in our metallicity range. On the other hand, $\mathrm{Mg}$ abundances should be reduced by 0.04 dex when derived from the $\mathrm{Mg}$ I line at $5528 \AA$ and by $0.03 \mathrm{dex}$ for the two $\mathrm{Mg}$ Ib lines (Zhao et al. 1998). This would lead to a systematic overestimate of $[\mathrm{Mg} / \mathrm{Fe}]$ of $0.33 \mathrm{dex}$ in LTE (with a $0.04 \mathrm{dex}$ difference in the NLTE corrections between the lowest and the highest metallicity of our stellar sample). However, since Fe is mostly present in its ionized state, Fe abundances derived from Fe II lines are insensitive to overionization, and a higher value of $\log (g)_{\text {NLTE }}$ (by 0.3-0.4 dex following Thévenin \& Idiart 1999) would be required to obtain ionization balance. This would further increase the overestimate of $[\mathrm{Mg} / \mathrm{Fe}]$ to $0.45 \mathrm{dex}$ in LTE.

It is important to note that Gratton et al. (1999) found different results when calculating NLTE corrections to Fe and $\mathrm{Mg}$ abundances. They suggest corrections that are very small, if not negligible, for main-sequence and RGB stars. This is in conflict with the calculations of Thévenin \& Idiart (1999), leaving NLTE corrections still very uncertain.

The assumption of LTE is thus presumably introducing errors in our abundances but, because of the homogeneity of our sample and the small variation of NLTE corrections on the metallicity range considered, we assume any effect on $[\mathrm{Mg} / \mathrm{Fe}]$ is buried in our random uncertainties. Furthermore, because of the present uncertainties on the effects of NLTE, and in order to have results comparable with most observational studies, we adopt the LTE estimates in our discussion, warning the reader that our $[\mathrm{Mg} / \mathrm{Fe}]$ ratio may be overestimated.

\subsection{Abundance error estimates}

In Sect. 3.5 we estimated the uncertainties on the adopted stellar parameters. In Table 5 we showed the dependence of the abundances on these parameters taken one at time; in Table 6 the results of the Monte Carlo test showed the combined dependence on iteratively determined $\log (g)$ and $\xi$ values to have a smaller impact on the abundances than the quadratic sum of the two errors taken separately. Moreover, the Monte Carlo test was performed propagating numerically the uncertainty on the $E W$, thus including the contribution of the latter in the result. We can now estimate the errors on our final abundances. We treat separately the random errors that affect the scatter of our results, and the systematic errors that affect the absolute values (and thus the average $[\mathrm{Mg} / \mathrm{Fe}]$ ).

\subsubsection{Random errors}

As shown in Table 6 , the uncertainty on the $E W$ propagates through the determination of the parameters $\log (g)$ and $\xi$, and 
affects the resulting $[\mathrm{Fe} / \mathrm{H}]$ by $0.02 \mathrm{dex},[\mathrm{Mg} / \mathrm{H}]$ by up to $0.06 \mathrm{dex}$, and $[\mathrm{Mg} / \mathrm{Fe}]$ by up to $0.06 \mathrm{dex}$. We could adopt these values as the uncertainties on the abundances due to the combination of the three factors, $1 \sigma_{A}\left(\sigma_{E W}, \sigma_{\log (g)}, \sigma_{\xi}\right)$. However, we make use of $\log (g)$ values from the photometric calibration, hence the Monte Carlo test does not reflect the entire procedure. We are obliged to estimate our errors as the quadratic sum of the uncertainty due to single parameter variations, and consider the results of the Monte Carlo test as a conservative estimate of the error due to uncertainty on line measurement, $\sigma_{A}\left(\sigma_{E W}\right)$.

Adding the random uncertainty on temperature, $\xi$ and $\log (g)$ (Table 5) increases the uncertainty on the abundances to 0.04 dex for $[\mathrm{Fe} / \mathrm{H}], 0.10$ dex for $[\mathrm{Mg} / \mathrm{H}]_{\mathrm{Ib}}$ and 0.08 dex for $[\mathrm{Mg} / \mathrm{Fe}]_{\mathrm{Ib}}$. Stars where the weak $\mathrm{Mg} 5528 \AA$ line was not measured have a slightly higher uncertainty on $\mathrm{Mg}$, by about $0.01 \mathrm{dex}$.

$\log (g f)$ values have been chosen on the basis of an experimental determination with uncertainties $<25 \%$. This implies that we have to account for a corresponding $<0.1$ dex uncertainty in the abundances derived from single lines. Having a number of lines $N$ will reduce the uncertainty in the average by a factor $1 / \sqrt{N}$. For $\mathrm{Fe}$ abundances, where the number of lines is statistically significant, we assume the standard error of the $\mathrm{Fe}$ abundance averaged on all the lines to be a good estimate of the total random error. This is up to 0.03 dex. For $\mathrm{Mg}$, the two or three lines give a random error due to $g f$-values of $\sim 0.06 \mathrm{dex}$. However, in the case of $\mathrm{Mg}$, we treat these errors as systematic, as described in the next section. Adding these uncertainties quadratically to the previous estimates produces a total random error of $0.05 \operatorname{dex}$ for $[\mathrm{Fe} / \mathrm{H}], 0.10 \mathrm{dex}$ for $[\mathrm{Mg} / \mathrm{H}]$ and 0.08 dex for $[\mathrm{Mg} / \mathrm{Fe}]$.

A final remark is relevant on the uncertainties derived by RNB on their temperature estimates. Since their $40 \mathrm{~K}$ random uncertainty is very low, and our $[\mathrm{Fe} / \mathrm{H}]$ abundances are very sensitive to temperature, we explored the effect of adopting an artificially more conservative $\sigma=80 \mathrm{~K}$ error for this study. This would increase the random uncertainty on $[\mathrm{Fe} / \mathrm{H}]$ to $0.07 \mathrm{dex}$ and that on $[\mathrm{Mg} / \mathrm{H}]$ to $0.11 \mathrm{dex}$. The $[\mathrm{Mg} / \mathrm{Fe}]$ error remains unchanged at $0.08 \mathrm{dex}$.

\subsubsection{Systematic errors}

We estimate the effect of systematic errors in our model parameters as follows. We consider the effect of an overestimate of $150 \mathrm{~K}$ as the systematic error on the temperature, based on the previous discussion of the photometric estimate adopted in this study - see Sect. 3.5. The variation on $[\mathrm{Mg} / \mathrm{Fe}]$ for a systematic change of $-150 \mathrm{~K}$ alone would be less than $-0.01 \mathrm{dex}$, because of an equivalent decrease of $\sim 0.14 \mathrm{dex}$ in both $[\mathrm{Fe} / \mathrm{H}]$ and $[\mathrm{Mg} / \mathrm{H}]$. However, the procedure used to determine the spectroscopic $\log (g)$ would partially compensate for the $T_{\text {eff }}$ change, resulting in a decrease in $\log (g)$ of about $0.3 \mathrm{dex}$, leading to an overall increase of $0.04 \mathrm{dex}$ for $[\mathrm{Mg} / \mathrm{H}]$. The overall variation on $[\mathrm{Fe} / \mathrm{H}]$ is $0.12 \mathrm{dex}$, because of the much weaker sensitivity to changes in $\log (g)$. This produces a 0.08 dex change in $[\mathrm{Mg} / \mathrm{Fe}]$, because of the greater sensitivity of $\mathrm{Mg}$ Ib lines to $\log (g)$.
The uncertainty on $g f$-values can be a large contribution to our uncertainty on the abundances, and requires further comment. For $\mathrm{Fe}$, we introduced the uncertainties on the $g f$-values in the random error. However, in the case of the $\mathrm{Mg} \mathrm{Ib}$ lines, the uncertainty on the $g f$-values should be treated like a systematic effect, since the two $\mathrm{Mg}$ Ib lines are averaged only with the weak Mg line at $5528 \mathrm{~m} \AA$. Because of the similar EPs and strength of the two Mg Ib lines, errors in the $\mathrm{Mg} g f$-values could cause the same variation in the line abundances of both, thus shifting our $\mathrm{Mg}$ abundances by the same amount. This will affect the mean value of the $\mathrm{Mg}$ abundance of a star but not the line-to-line scatter about the mean. We prefer to include the uncertainty in the $\mathrm{Mg} g f$-values separately from the random error derived in the previous subsection. If we do so, the random error on $[\mathrm{Mg} / \mathrm{Fe}]$ is 0.08 dex, while the systematic error becomes the sum of the systematic error due to temperature $(0.08 \mathrm{dex})$ and the one due to the $g f$-values $(0.06 \mathrm{dex})$, i.e. the total systematic uncertainty on $[\mathrm{Mg} / \mathrm{Fe}]$ is then 0.10 dex. The total systematic error for $[\mathrm{Mg} / \mathrm{H}]$ is $0.07 \mathrm{dex}$, while $[\mathrm{Fe} / \mathrm{H}]$ is affected systematically only by the temperature, i.e. by $0.12 \mathrm{dex}$.

\subsection{Metallicity comparison}

Figure 3 shows a comparison of the metallicity estimates of this study with the 1 A estimates of RNB (filled dots). The $1 \AA$ estimates were obtained by a calibration of the pseudo-equivalent width of the $\mathrm{Ca}$ II $\mathrm{K}$ line as a function of $B-V$ colour to indicate the stellar metallicity $[\mathrm{Fe} / \mathrm{H}]$ (Beers et al. 1999). A linear fit $a x+b$ gives $a=1.05$ and $b=0.23$, with an rms of $0.14 \mathrm{dex}$, larger than our uncertainties on $\mathrm{Fe}(0.05)$, but consistent with the value of 0.15 dex claimed by RNB for the $1 \AA$ estimates. In addition, we show the trend of Fe literature values as discussed by RNB (empty squares). A linear fit gives $a=0.96$ and $b=-0.16$, with a smaller scatter about the fit, $\mathrm{rms}=0.11 \mathrm{dex}$. This implies a slightly better agreement of our metallicity determination with previous literature determinations than with the $1 \AA$ estimates. Both, however, can be considered in good agreement with our estimates within the stated uncertainties.

\section{Discussion}

\subsection{Fe and $\mathrm{Mg}$ abundances}

The abundances of Fe and $\mathrm{Mg}$ are shown in Table 3. $[\mathrm{Mg} / \mathrm{Fe}]$ is within a range of $0.20 \mathrm{dex}$, between 0.22 and 0.42 . Figure 4 displays the run of $[\mathrm{Mg} / \mathrm{Fe}]$ vs. $[\mathrm{Fe} / \mathrm{H}]$. The thick line is the result of a linear least squares fit and the two thin lines delimit a $\pm 1 \sigma$ region, $\sigma=0.06$ dex being the standard deviation of points about the fit. The equation of the linear fit is:

$[\mathrm{Mg} / \mathrm{Fe}]=(0.00 \pm 0.04) \cdot[\mathrm{Fe} / \mathrm{H}]+(0.31 \pm 0.12)$

The result of this linear fit suggests that the trend of $[\mathrm{Mg} / \mathrm{Fe}]$ as a function of $[\mathrm{Fe} / \mathrm{H}]$ is flat. We calculate a weighted mean for $[\mathrm{Mg} / \mathrm{Fe}]$, the weights being the inverse of the square of the adopted uncertainties. The weighted average, standard error and standard deviation are

$\overline{[\mathrm{Mg} / \mathrm{Fe}]}=0.30 \pm 0.01 \quad \sigma=0.06$. 


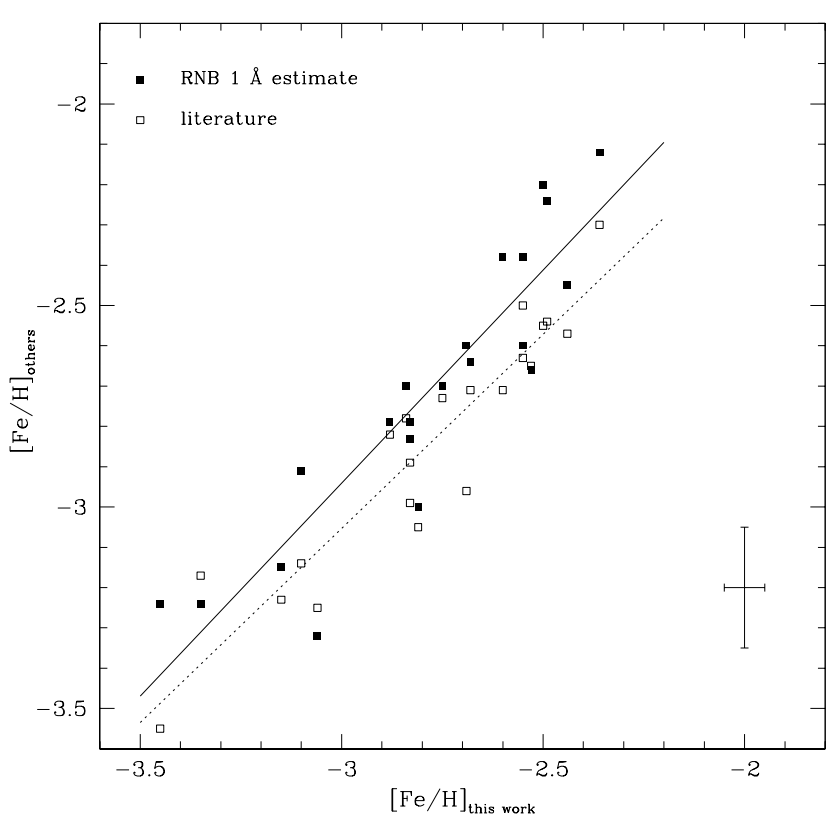

Fig. 3. A comparison between the Fe estimates of this study and the $1 \AA$ (filled squares) and literature (empty squares) estimates respectively. Overplotted are the two linear fits.

Systematic errors are not accounted for in this statement; they may affect the mean value by up to $0.10 \mathrm{dex}$, which we take to be the limit on the precision with which the average value of $[\mathrm{Mg} / \mathrm{Fe}]$ is determined.

The scatter is not affected by systematic errors, hence, if no real intrinsic scatter was present, we would expect the rms scatter $(0.06 \mathrm{dex})$ to be no larger than the random uncertainty $(0.08 \mathrm{dex})$, as is the case. Moreover, the standard deviation of our results is similar to that of the Monte Carlo simulation that was performed starting from the data of a single star (but which excluded random errors in $\left.T_{\text {eff }}\right)$. We thus infer that the dispersion in the results is due to our analysis; any intrinsic scatter in $[\mathrm{Mg} / \mathrm{Fe}]$ of the sample must be much smaller, $\ll 0.06$ dex. This is our most important result, and is a considerable reduction on the Cayrel et al. standard deviation on $[\mathrm{Mg} / \mathrm{Fe}]$, 0.13 dex. We consider the implications in Sect. 5.3.

The very low scatter confirms that our selection criteria and analysis produced a very homogeneous sample. A higher scatter could have been the signature of an intrinsic characteristic of the stellar sample or star-to-star differences introduced by the analysis. The latter can be difficult to estimate because of its dependence on the adopted model atmospheres. As an example of the difficulties in quantifying the real scatter, Johnson's (2002) study of giants has a dependence of $[\mathrm{Mg} / \mathrm{Fe}]$ on both $\log (g)$ and $T,[\mathrm{Mg} / \mathrm{Fe}]$ showing a very low scatter in the run versus each parameter but a high scatter versus $[\mathrm{Fe} / \mathrm{H}]$. A similar problem is buried in the results of Cayrel et al. (2003) whose mean, $[\mathrm{Mg} / \mathrm{Fe}]=+0.27 \mathrm{dex}$, is in reasonable agreement with our findings, but their standard deviation, 0.13 dex, has a component due to a slight dependence of their abundances on temperature and gravity. Our utilisation of turnoff stars covering only a narrow range of temperatures and surface gravity has avoided this difficulty. In less homogeneous studies, different stellar evolutionary stages are considered in the same sample, thus including a star-to-star difference in the dependence on the adopted stellar atmospheres. Finally, the use of similar absorption lines for all the stars has limited the influences of uncertainties in $g f$-values to a systematic uncertainty on the average $[\mathrm{Mg} / \mathrm{Fe}]$, not affecting the scatter. Further studies of larger, critically-selected samples will aid in determining the intrinsic properties of the Galaxy.

\subsection{Other elements}

The abundances derived for the other elements are shown in Table 4 based on up to five lines of CaI, three of Ti I and three of $\mathrm{Cr}$ I. The trends of these abundances are shown in Fig. 4.

For $[\mathrm{Ca} / \mathrm{Fe}]$, we show a linear least square fits as a function of $[\mathrm{Fe} / \mathrm{H}]$. A statistical test indicates that the slope is not significant. Since we have Ca estimates for all but two stars of our sample (CD-24 17504 and LP831-70), we use [Ca/Fe] to verify the consistency of our $\alpha$-element abundances. The mean $[\mathrm{Ca} / \mathrm{Fe}]$ is +0.37 , with a slightly higher standard deviation than for $[\mathrm{Mg} / \mathrm{Fe}], 0.09 \mathrm{dex}$. The estimated random error on $[\mathrm{Ca} / \mathrm{Fe}]$ is slightly lower than the one on $[\mathrm{Mg} / \mathrm{Fe}]$ - on average $0.07 \mathrm{dex}$. (The Monte Carlo simulation, which excludes the temperature and the photometric calibration for the gravity, showed a much smaller error for $[\mathrm{Ca} / \mathrm{Fe}]$, of order 0.02 dex, because of the smaller sensitivity of the weak lines used for $\mathrm{Ca}$ to changes in stellar parameters.) Consequently, we cannot rule out the existence of an intrinsic scatter in the $[\mathrm{Ca} / \mathrm{Fe}]$ values up to $\sigma \sim$ 0.06 dex.

Previous authors have used an $\alpha$-index averaging $\mathrm{Mg}$ and Ca chemical abundances (see e.g., Gratton et al. 2000). However, the Thielemann et al. (1996) SN II models show that $\mathrm{Ca}$ yields are only slightly dependent on progenitor mass, whereas $\mathrm{Mg}$ yields depend quite strognly on progenitor mass. This leads iGCE models to predict a lower scatter for Ca than for $\mathrm{Mg}$ (Argast et al. 2000). Moreover, Ca is synthesised deeper in the progenitor than $\mathrm{Mg}$, so Ca yields are dependent also on the energy of the explosion, and hence not as independent as possible of SN II model uncertainties. We therefore concentrate on $[\mathrm{Mg} / \mathrm{Fe}]$.

We also tabulate abundances for $\mathrm{Cr}$ and $\mathrm{Ti}$. $\mathrm{Cr}$ absolute abundances are, on average, similar to those of Fe. We confirm the well-known overabundance of Ti relative to Fe, though with a suspicious dependence on $[\mathrm{Fe} / \mathrm{H}]$. However, the Ti abundances have been poorly determined due to the use of only one line for most of the stars, and hence we place little weight on this result. We do not investigate this element further.

\subsection{Implications for Galactic chemical evolution}

\subsubsection{Restatement of the problem}

The absence of intrinsic scatter in the $\mathrm{Mg}$ abundances presents a real challenge for inhomogeneous Galactic chemical evolution models. Argast et al. (2000, 2002) and Tsujimoto \& Shigeyama (1998) have shown that iGCE models predict a large scatter in $[\mathrm{Mg} / \mathrm{Fe}]$ at $[\mathrm{Fe} / \mathrm{H}]<-2.5$ that reflects the dependence of nucleosynthesis on the progenitor mass of individual SN II, at a time in the history of GCE when the ISM 

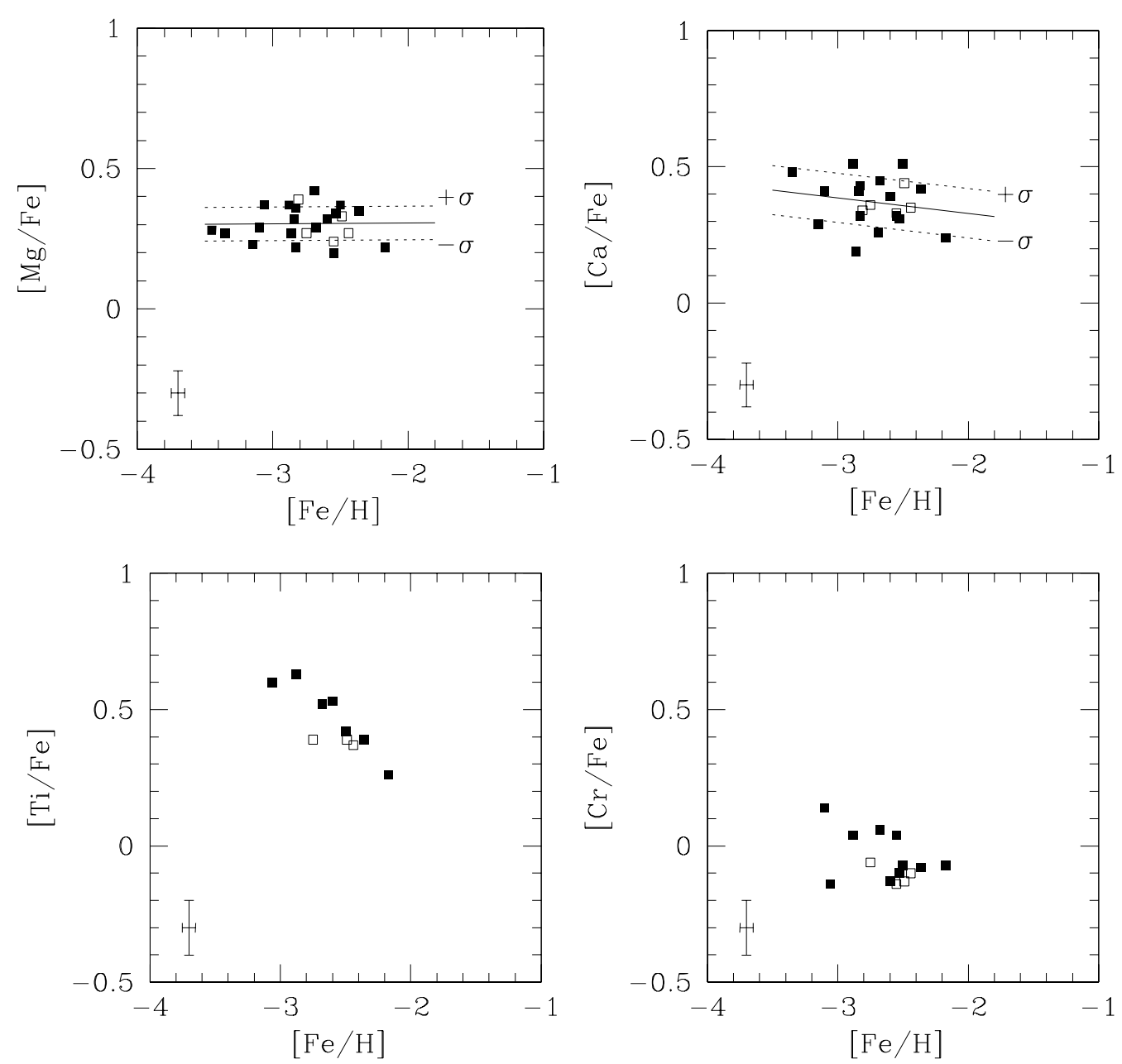

Fig. 4. Abundances are shown as $[\mathrm{el} / \mathrm{Fe}]$ vs. $[\mathrm{Fe} / \mathrm{H}]$. Empty squares are stars with no $\mathrm{Fe}$ II measurements. In the case of $\mathrm{Mg}$ and $\mathrm{Ca}$, we show the results of a linear fit (thick line) and the $\pm 1 \sigma$ region. This is not shown for $\mathrm{Ti}$ and $\mathrm{Cr}$ because of the small number of stars with $\mathrm{Ti}$ and Cr measurements.

was poorly mixed. The François et al. (2004) homogeneous GCE model can reproduce the abundance patterns observed in the most recent observations by requiring a large correction to SN II yields. One has to investigate why homogeneity occurs in a metallicity region expected to be inhomogeneous.

The Argast et al. model predicts that for $[\mathrm{Fe} / \mathrm{H}]<-3.0$, SN II pollute only locally, i.e., the ISM is mixed at a rate slower than successive SN II events, and individual SN yields are reflected by the local ISM. In the range $-3.0<[\mathrm{Fe} / \mathrm{H}]<$ -2.0 , mixing of the ISM causes the first overlaps of polluted regions, though some regions that are unenriched remain. At $[\mathrm{Fe} / \mathrm{H}]=-2.5$, some regions of the ISM are enriched several times by supernova progenitors of different masses. One should thus observe stars showing abundance patterns characteristic of SN progenitors of different masses. By $[\mathrm{Fe} / \mathrm{H}]>$ -2.0 , the yields from successive polluting events are averaged over the initial mass function (IMF) and well mixed, bringing iGCE and 1-zone GCE models into agreement. Our metallicity range $(-3.4<[\mathrm{Fe} / \mathrm{H}]-2.2)$ covers the inhomogeneous part of the ISM history, where a range up to 1 dex is predicted for $\mathrm{Mg}$ abundances, compared to a range of 0.2 dex for our observations.
In Figs. 5 and 6, we show the standard deviation of Argast's model calculated for 0.1 dex metallicity bins. The two figures correspond to the models shown in Figs. 1 and 14 of Argast et al. (2002), calculated adopting SN II yields from Thielemann et al. (1996), and from the Argast et al. "H1" empirical model, respectively. The yields from Thielemann et al. (1996) and Nomoto et al. (1997), adopted in the Argast et al. model, range from $[\mathrm{Mg} / \mathrm{Fe}]=-0.83$ for a progenitor of $13 M_{\odot}$ to $[\mathrm{Mg} / \mathrm{Fe}]=$ 1.44 for a progenitor of $70 M_{\odot}$, with a monotonic relationship between the Mg mass yield and the progenitor mass. The H1 model was obtained by modifying the SN II yields to match the Fe yields inferred from SN II observations, rather than using yields from a SN II model, and was further constrained by the range of abundances previously observed in low-metallicity stars. This model shows a much lower scatter due to a smaller adopted range in $\mathrm{SN}$ II $\mathrm{Mg} / \mathrm{Fe}$ yield ratios. Superimposed on these figures are shown our standard deviation for $[\mathrm{Mg} / \mathrm{Fe}]$, and the predicted mean standard deviations of the models calculated over the same metallicity range. We also show the standard deviation of $[\mathrm{Mg} / \mathrm{Fe}]$ from Cayrel et al. (2003). For the $\mathrm{H} 1$ model, the predicted standard deviation in $[\mathrm{Mg} / \mathrm{Fe}]$ is $\sim 0.17$ dex, rising towards lower metallicities, and well in excess of our limit of 0.06 dex. 


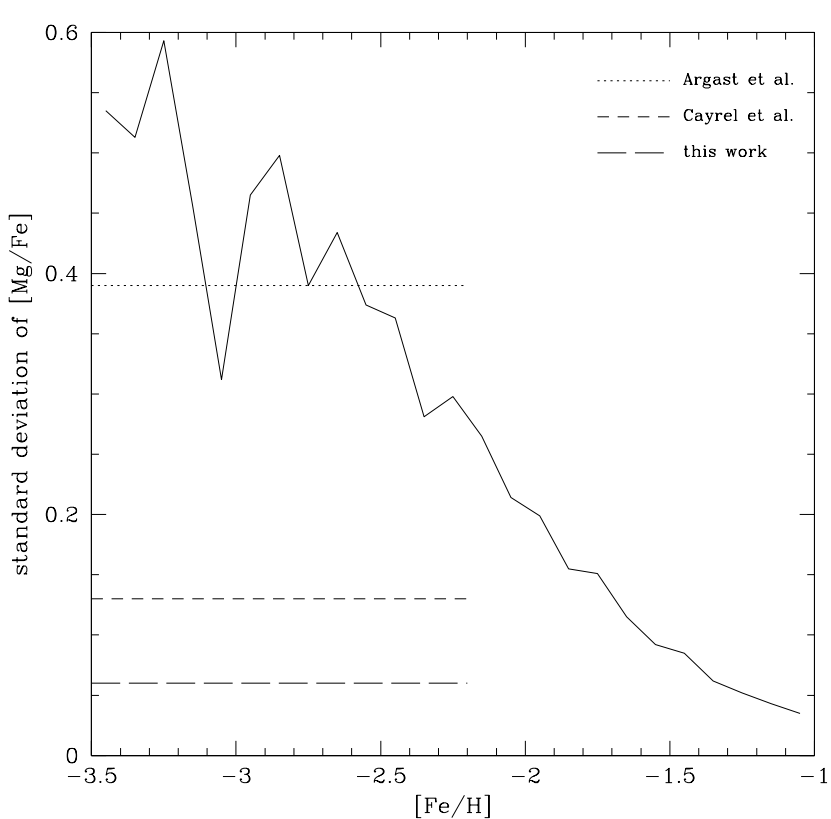

Fig. 5. The standard deviation of the dispersion in $[\mathrm{Mg} / \mathrm{Fe}]$ predicted by the model of Argast et al. (2002, their Fig. 1), in the case of SN II yields adopted from Thielemann et al. (1996). The dotted, small dashed and the dashed lines show the standard deviation over the metallicity range of our observations, $-3.4<[\mathrm{Fe} / \mathrm{H}]<-2.2$, respectively for Argast et al. (2002), Cayrel et al. (2003) and this work.

Theoretically (Arnett 1996), the assumption of instantaneous mixing adopted by 1-zone GCE models is not correct at these low metallicities, hence inhomogeneity must be considered in the models. If iGCE models are correct in relaxing the instantaneous mixing assumption, then the lack of scatter in the $[\mathrm{Mg} / \mathrm{Fe}]$ values we have measured is all the more remarkable. To explain this result, one has to focus on SN II yields and other uncertainties in the models.

\subsubsection{A restricted mass range for $\mathrm{SN}$ II progenitors}

Chiappini et al. (1999) found that, for metallicities $-3.0<$ $[\mathrm{Fe} / \mathrm{H}]<-1.0$, the trends in $\alpha$-elements are expected to show slopes. This is not seen in our flat trend at $[\mathrm{Fe} / \mathrm{H}]<-2.2$. However, this slope is not predicted in other models (e.g. Argast 2002). The uncertainty on our measurement of the slope restricts the variation of the $[\mathrm{Mg} / \mathrm{Fe}]$ slope to within $0.04 \mathrm{dex}$ from flat over the metallicity range $-3.0<[\mathrm{Fe} / \mathrm{H}]<-2.0$.

The range of progenitor masses that give rise to $[\mathrm{Mg} / \mathrm{Fe}]=$ $0.3 \pm 0.1$ (our observational value) is $18-20 M_{\odot}$ according to the yields of Thielemann et al. (1996). However, even if we knew the exact yields of this progenitor mass range, the flat trend and the lack of intrinsic scatter raises the question: why, at any time in the early Galaxy, would the only contribution to the ISM chemical composition of Mg come from this narrow range of progenitors?

The upper mass limit that avoids complete collapse to a black hole is unknown, and while it is believed to be in the range of $30-50 M_{\odot}$, there is some possibility it could be lower in low-metallicity stars. Maeder (1992) found that a limit around 20-25 $M_{\odot}$ was consistent with $\Delta Y / \Delta Z$ (the enrichment

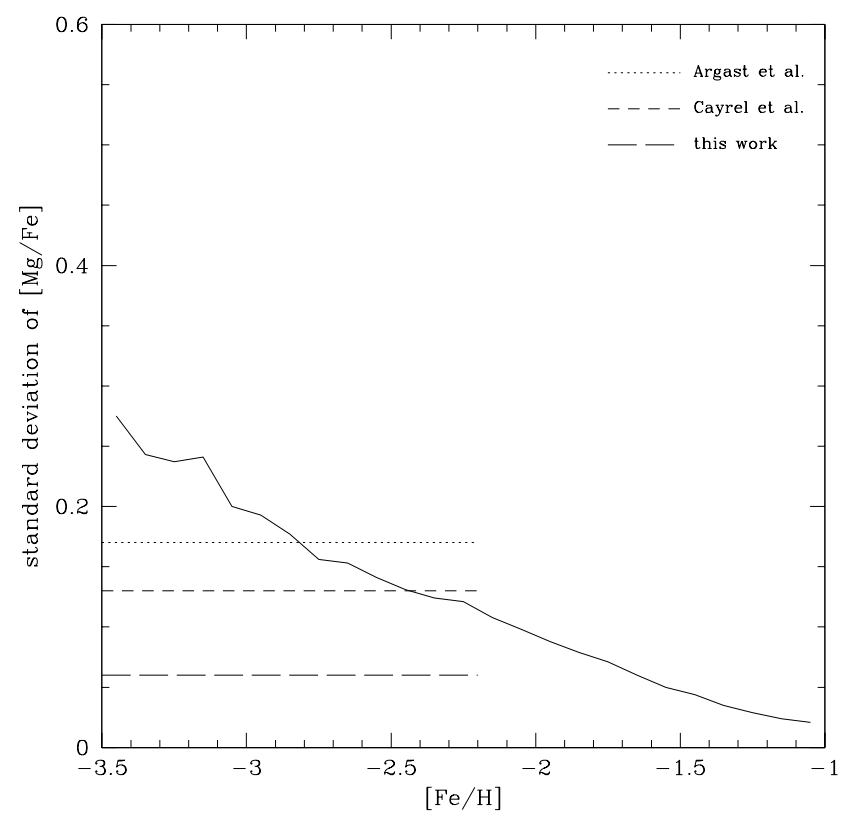

Fig. 6. The standard deviation of the dispersion in $[\mathrm{Mg} / \mathrm{Fe}]$ predicted by the model of Argast et al. (2002, their Fig. 14), in the case of their H1 model. The dotted, small dashed and the dashed lines show the standard deviation over the metallicity range of our observations, $-3.4<[\mathrm{Fe} / \mathrm{H}]<-2.2$, respectively for Argast et al. (2002), Cayrel et al. (2003) and this work.

of helium relative to metals). More recently, Fryer (1999) confirmed the $20 M_{\odot}$ limit for the initial mass for black hole formation. Fryer \& Heger (2000) suggested that stellar wind and rotation could decrease the energy of the explosion and thus the lower mass limit for black hole formation.

However, even if the upper mass limit for $\mathrm{Mg}$ enrichment of the ISM could be $20 M_{\odot}$, there is no reason to think that progenitors as low as 10-12 $M_{\odot}$ would be disabled. One may question our theoretical understanding of SN II models, or look for criteria that would favour SN II with this progenitor mass. One possibility is a shallower (top-heavy) IMF in the early phases to raise the production of massive stars, allowing progenitors near the black-hole mass limit $\left(20 M_{\odot}\right.$ ?) to dominate enrichment of the ISM. $10 M_{\odot}$ SN II progenitors would nevertheless still evolve within $\sim 30 \mathrm{Myr}$, which is the timescale for the enrichment of the halo to $[\mathrm{Fe} / \mathrm{H}]=-3.0$ according to models which explain the rise of the r-process enrichment in $[\mathrm{Ba} / \mathrm{Fe}]$ and $[\mathrm{Eu} / \mathrm{Fe}]$ in the halo in terms of low mass $\left(8-10 M_{\odot}\right)$ SN II (Mathews \& Cowan 1990; Travaglio et al. 2001; Ishimaru et al. 2004).

On balance, we conclude that currently there is no support for the proposition that only a narrow range of SN II progenitor masses around $18-20 M_{\odot}$ contributes significantly to the Galactic enrichment in $\mathrm{Mg}$.

\subsubsection{SN II yields}

The iron yields from SN II are notoriously difficult to predict (Nomoto et al. 1997); Thielemann et al. (1996) assume a decline of Fe with rising progenitor mass, while Woosley $\&$ Weaver (1996) assume an increase. Given this uncertainty, 
could the majority of SN II produce the same $[\mathrm{Mg} / \mathrm{Fe}]$ ratio in their ejecta? $\mathrm{A} \mathrm{Mg} / \mathrm{Fe}$ yield ratio constant with progenitor mass (and thus constant over the history of the ISM) would require a proportionality between the size of the ejected $\mathrm{Si}$ burned shell (the source of $\mathrm{Fe}$ ) and the mass of the progenitor (which correlates with $\mathrm{Mg}$ yield). It seems highly unlikely that nature should achieve such a perfect balance in the hydrostatic production of $\mathrm{Mg}$ and the subsequent ejection of a fraction of the explosive production of Fe, across the $\sim 10-20$ or $10-30 M_{\odot}$ mass range of SN II progenitors. Moreover, the flat slope found for $[\mathrm{Ca} / \mathrm{Fe}]$ versus metallicity would be affected by a change in the Fe yields, as would other element $[\mathrm{el} / \mathrm{Fe}] \mathrm{ratios}$, and the $\mathrm{Ca}$ yield would also depend on explosion energy and progenitor mass. Thielemann's models do not show a variation of $\mathrm{Ca}$ with progenitor mass of the same order as that of $\mathrm{Mg}$.

Attempts to reproduce the chemical pattern of very lowmetallicity stars by adjusting the mass cut in SN II explosion models have been unsuccessful (Chieffi \& Limongi 2002). Tsujimoto \& Shigeyama (1998) traced yield versus mass for SN II from the observations of McWilliam et al. (1995) and the yields from Woosley \& Weaver (1996), and satisfactorily produced predictions for averaged abundances ratios within 0.1 dex of the observations. Their final remark is the need of revising SN II yields of elements except $\mathrm{C}, \mathrm{O}$, and $\mathrm{Mg}$. Our results strongly indicate that either also $\mathrm{Mg}$ yields are wrong (see also Argast et al. 2002), and Fe yields are wrong, or the theoretical framework of iGCE has reached its limit and needs a deeper understanding of mixing.

François et al. (2004) have shown that constraints to SN II yields can be found by matching observations with their homogeneous GCE model. However, even if SN II yields are revised following their prescription, within the framework of homogeneous models, the lack of scatter still remains to be explained. If $[\mathrm{Mg} / \mathrm{Fe}]$ yields truly are not constant for all progenitor masses, and there is not only a very narrow mass range of $\mathrm{SN}$ II progenitors that produce significant $\mathrm{Mg}$, then the lack of scatter points to the following unexpected conclusion: the ISM from which stars formed at $[\mathrm{Fe} / \mathrm{H}]>-3.5$ was on the whole already well-mixed, and it was enriched by a sufficient number of SN II to show an IMF averaged pattern at $[\mathrm{Fe} / \mathrm{H}]<-3.0$, in spite of the expectations to the contrary from iGCE current models. Furthermore, the hypothesis by Cayrel et al. (2003) of the existence of a plateau in the $[\mathrm{el} / \mathrm{Fe}]$ ratios at very low metallicity is clearly supported by our $[\mathrm{Mg} / \mathrm{Fe}]$ ratio. We are left to puzzle over how this IMF average is achieved ove much of the halo on the short timescales ( $\leq 30 \mathrm{Myr}$ ) for the ejection of the full range of SN II progenitor masses $\left(M \geq 10 M_{\odot}\right)$. This could imply that we are observing the primordial abundances from the first stars below $[\mathrm{Fe} / \mathrm{H}]=-3.0$, as Cayrel et al. suggested.

\subsubsection{Mixing and cooling timescales}

Enrichment timescales are not well constrained. Prantzos (2003) shows that an early phase of infall and relaxing the instantaneous recycling approximation causes his halo outflow model to reproduce better the metallicity distribution of stars in the halo. Enrichment occurs much quicker in his model than in iGCE models, but the introduction of an early infall phase in his model slows the metallicity enrichment again, as a result of which the time needed to reach $[\mathrm{Fe} / \mathrm{H}]=-3.0$ increases from $35 \mathrm{Myr}$ to $100 \mathrm{Myr}$. A similar delay in the enrichment could occur in iGCE models for a strong early infall phase. However, a slower enrichment of the Galaxy does not affect the predicted scatter in $[\mathrm{Mg} / \mathrm{Fe}]$ vs. $[\mathrm{Fe} / \mathrm{H}]$, but rather the agemetallicity relation.

De Avillez \& Mac Low (2002) studied the mixing timescale in a SNe-driven ISM, and show that inhomogeneities due to single SN II explosions take up to $350 \mathrm{Myr}$ to be erased (for the present Galactic SN II rate). Even when mixing scales as small as a few kpc are considered, efficient mixing requires of order 120 Myr. Increasing the SN rate by a factor 10, they find that inhomogeneities take only some tens of Myr to disappear. If increasing the SN II rate reduces the time needed for efficient mixing, then for a short time ( $30 \mathrm{Myr})$ it favours the explosion of higher mass progenitors (because of their shorter lifetimes) and a higher average $[\mathrm{Mg} / \mathrm{Fe}]$ ratio. This effect could also be achieved with a top-heavy IMF (discussed above). This would avoid the ISM having regions with low $[\mathrm{Mg} / \mathrm{Fe}]$ values, but would still require a progenitor upper mass limit of $20 M_{\odot}$ to avoid the production of $[\mathrm{Mg} / \mathrm{Fe}]>0.4$ except for at the very lowest metallicities (Norris 2003).

We asked above how an IMF-averaged $[\mathrm{Mg} / \mathrm{Fe}]$ value could be achieved on the very short evolutionary timescales of stars at $[\mathrm{Fe} / \mathrm{H}]<-3.0$. This may be the wrong question to ask if cooling timescales crucial to star formation, rather than mixing timescales, are the critical factor. iGCE models lack reliable treatments of the cooling of hot SN II ejecta in a metal-poor environment. If the cooling time exceeds the evolutionary time of the lowest mass SN II progenitors of $10 M_{\odot}$, i.e. $30 \mathrm{Myr}$, then a complete IMF-average of the $[\mathrm{Mg} / \mathrm{Fe}]$ ratio will be achieved, not on the evolutionary time of the SN II, but on the cooling timescale of the ISM, and therefore on the formation timescale of the next stellar generation.

Oey (2003) investigates mixing and cooling processes in the ISM by means of her simple inhomogeneous model (SIM) of Galactic chemical evolution, which explicitly incorporates interstellar mixing and mass transport. As previously found by De Avillez \& Mac Low (2002), diffusion is inefficient compared to turbulent mixing. Even though "turbulent processes are extremely difficult to constrain", turbulent mixing is found to be extremely efficient in the case of a hot ionized medium (HIM). Thus, in a phase of HIM-dominated ISM, efficient mixing could indeed occur. Furthermore, a hot ISM has a low cooling efficiency, thus a two-phase ISM could experience a delay due to the cooling time between the SN II events and the incorporation of their ejecta in star forming regions. Once new stars could form from SN-enriched gas, they would form out of a well- mixed ISM. By a comparison with the observed metallicity distribution function and the lowest metallicity observed in stars $([\mathrm{Fe} / \mathrm{H}]=-4.0$ at the time of Oey's analysis $)$, Oey's points out that an inhomogeneous phase could have been extremely short lived and that the ISM would have then mixed efficiently. Furthermore, Recchi et al. (2001) find that, in the case of dwarf galaxies, a single sturbust leads to the development of galactic winds and to a quick mixing. However, dwarf galaxies 
evolve in a low gravitational potential and thus dynamical processes (such as galactic wind) may have larger influences on the mixing than in the case of the Galaxy.

We believe further investigations of mixing and cooling timescales in relation to iGCE models are advisable, since there is no clear theoretical expectation that the problem of the missing inhomogeneities will be solved by adjustments of the SN II yields, the SN II progenitor mass range, or modification of the IMF.

\section{Summary}

We have presented the results of the chemical analysis of a sample of 23 main-sequence turnoff halo stars previously selected by RNB. Because of the small number of lines identified in the most metal-poor stars, the $[\mathrm{Mg} / \mathrm{Fe}]$ abundance ratio was determined on the basis of photometric calibrations for both effective temperature and surface gravity. This method should avoid introducing star-to-star differences and preserve the homogeneity of sample.

Our results show that $[\mathrm{Mg} / \mathrm{Fe}]$ is constant over the metallicity range considered. There is no indication of internal scatter, the 0.06 dex standard deviation about the mean being well within the expected 0.08 dex random uncertainty. The maximum point-to-point range of $[\mathrm{Mg} / \mathrm{Fe}]$ is only 0.2 dex.

The observed lack of scatter presents a challenge for iGCE models that predict a dispersion for $[\mathrm{Mg} / \mathrm{Fe}]$ at low metallicity, with standard deviation of the order 0.4 dex, and a total range as large as $1.0 \mathrm{dex}$. We review the possible sources of uncertainties in the iGCE models, such as the mass range of SN II progenitors and the $\mathrm{Mg}, \mathrm{Ca}$ and Fe yields, and the IMF, but find no obvious solution to the problem. We identify the need for exploring the time dependence of cooling and mixing in the early Galaxy, to verify whether these processes could cancel out the predicted scatter by ensuring that new stars form only once the enrichment of the ISM has been averaged over the IMF for the full range of SN II progenitor masses. This could occur during a hot early phase of the ISM, where turbulent mixing is extremely efficient in slowly cooling, hot, metal-deficient gas.

It is still difficult to reconcile the large spread in $[\mathrm{Sr} / \mathrm{Fe}]$ at $[\mathrm{Fe} / \mathrm{H}]<-2.0$ (discussed in Sect. 1) with the lack of a spread in $[\mathrm{Mg} / \mathrm{Fe}]$ found in Section 4. The latter argues for star formation occurring only after an IMF average of $\mathrm{Mg}$ yields has been achieved in the ISM, whereas the lack of uniformity in $[\mathrm{Sr} / \mathrm{Fe}]$ requires the existence of stochastic events that are not completely averaged out. If the source of $\mathrm{Sr}$ is more restricted in mass, or otherwise rarer than the source of $\mathrm{Mg}$ (which comes from the entire SN II range), then this apparent inconsistency might be resolved. The rise in $[\mathrm{Ba} / \mathrm{Fe}]$ toward $[\mathrm{Fe} / \mathrm{H}]=-2.5$, which may be due to the origin of r-process elements in 8-10 $M_{\odot}$ stars (Sect. 5.3.2) again argues against an IMF average being achieved by $[\mathrm{Fe} / \mathrm{H}]=-3.0$. However, if the $\mathrm{Mg}$ yield of such low mass stars is almost insignificant, as theory suggests, then the IMF average we infer from the Mg observations is probably a constraint only on higher mass SN II progenitors. (That is to say, the IMF average claimed for $\mathrm{Mg}$ is an average over most of the IMF at masses $M \sim 20 M_{\odot}$ rather than an average over strictly all of the SN II progenitor range.) The different behaviour of $[\mathrm{Ba} / \mathrm{Fe}]$ and $[\mathrm{Mg} / \mathrm{Fe}]$ emphasises, according to this interpretation, that $\mathrm{Ba}$ and $\mathrm{Mg}$ are predominantly synthesized in different progenitor mass ranges.

Acknowledgements. E.A. acknowledges Roberto Gallino for useful suggestions and discussions. D.A. acknowledges funding by the Swiss National Science Foundation. J.N.E. acknowledges funding by the Australia Research Council T.C.B. acknowledges partial funding for this work from grants AST 00-98508 and AST 00-98549, as well as from grant PHY 02-16783: Physics Frontiers Center/Joint Institute for Nuclear Astrophysics (JINA), awarded by the US National Science Foundation.

\section{References}

Allen, C., Schuster, W. J., \& Proveda, A. 1991, A\&A, 224, 280

Alonso, A., Arribas, S., \& Martínez-Roger, C. 1998, A\&AS, 139, 335

Anders, E., \& Grevesse, N. 1989, Geochim. Cosmochim. Acta, 53, 197

Anstee, S. D., O’Mara, B. J., \& Ross, J. E. 1998, Proc. IAU Symp. 189, on Fundamental Stellar Properties: The Interaction between Observation and Theory, ed. T. R. Bedding, A. J. Booth, \& J. Davis (Dordrecht: Kluwer), 179

Argast, D., Samland, M., Thielemann, F.-K., \& Gerhard, O. E. 2002, A\&A, 388, 842

Argast, D., Samland, M., Gerhard, O. E., \& Thielemann, F.-K. 2000, A\&A, 356, 873

Arnett, D. 1996, Supernovae and Nucleosynthesis (Princeton: Princeton University Press)

Audouze, J., \& Silk, J. 1995, ApJ, 451, L49

Bard, A., \& Kock, M. 1994, A\&A, 282, 1014

Bard, A., Kock, A., \& Kock, M. 1991, A\&A, 248, 315

Beers, T. C., Drilling, J. S., Rossi, S., et al. 2002, AJ, 124, 931

Beers, T. C., Rossi, S., Norris, J. E., Ryan, S. G., \& Shefler, T. 1999, AJ, 117, 981

Beers, T. C., Preston, G. W., \& Shectman, S. A. 1992, AJ, 103, 1987

Bell, R. A., \& Oke, J. B. 1986, ApJ, 307, 253

Bell, R. A., Eriksson, K., Gustafsson, B., \& Nordlund, A. 1976, A\&AS, 23, 37

Blackwell, D. E., Petford, A. D., \& Shallis, M. J. 1979, MNRAS, 186, 657

Blackwell, D. E., Petford, A. D., Shallis, M. J., \& Simmons, G. J. 1980, MNRAS, 191, 445

Blackwell, D. E., Menon, S. L. R., Petford, A. D., \& Shallis, M. J. 1982a, MNRAS, 201, 611

Blackwell, D. E., Petford, A. D., Shallis, M. J., \& Legget, S. 1982b, MNRAS, 199, 21

Blackwell, D. E., Petford, A. D., Shallis, M. J., \& Simmons, G. J. 1982c, MNRAS, 199, 43

Blackwell, D. E., Booth, A. J., Haddock, D. J., Petford, A. D., \& Leggett, S. K. 1986, MNRAS, 220, 549

Burstein, D., \& Heiles, C. 1982, AJ, 87, 1165

Carney, B. W., Latham, D. W., Laird, J. B., \& Aguilar, L. A. 1994, AJ, 107,2240

Carretta, E., Gratton, R., Cohen, J., Beers, E., \& Christlieb, N. 2002, AJ, 124, 481

Cayrel, R., Depagne, E., Spite, M., et al. 2003 [arXiv:astro-ph/0311082]

Chang, T. N. 1990, Phys. Rev. A, 41, 4922

Chang, T. N., \& Tang, X. 1990, J. Quant. Spectrosc. Radiat. Transfer, 43, 207 
Chen, Y. Q., Nissen, P. E., Zhao, G., Zhang, H. W., \& Benoni, T. 2000, A\&AS, 141, 491

Chiappini, C., Matteucci, F., Beers, T. C., \& Nomato, K. 1999, ApJ, 515,226

Chiba, M., \& Beers, T. C. 2000, AJ, 119, 2843

Chieffi, A., \& Limongi, M. 2002, ApJ, 577, 281

De Avillez, M. A., \& Mac Low, M.-M. 2002, ApJ, 581, 1047

Feltzing, S., Bensby, T., \& Lundström, I. 2003, A\&A, 397, L1

Ford, A., Jeffries, R. D., Smalley, B., et al. 2002, A\&A, 393, 617

François, P., Matteucci, F., Cayrel, R., et al. 2004 [arXiv: astro-ph/0401499]

Fryer, C. L. 1999, ApJ, 522, 413

Fryer, C. L., \& Heger, A. 2000, ApJ, 541, 1033

Fuhr, J. R., Martin, G. A., \& Wiese, W. L. 1988, J. Phys. Chem. Ref. Data, 17, Suppl. 4

Fuhrmann, K. 1998, A\&A, 338, 161

Fuhrmann, K., Axer, M., \& Gehren, T. 1995, A\&A, 301, 492

Golay, M. 1974, Introduction to astronomical photometry (Dordrecht: D. Reidel Publishing Co.), Astrophysics and Space Science Library, Vol. 41

Gratton, R. G., Carretta, E., Matteucci, F., \& Sneden, C. 2000, A\&A, 358,671

Gratton, R. G., Carretta, E., Eriksson, K., \& Gustafsson, B. 1999, ApJ, 350,955

Gratton, R. G., \& Sneden, C. 1994, A\&A, 287, 927

Grevesse, N., \& Sauval, A. J. 1998, Space Sci. Rev., 85, 161

Idiart, T., \& Thévenin, F. 2000, ApJ, 541, 207

Ibukiyama, A., \& Arimoto, N. 2002, A\&A, 394, 927

Ishimaru, Y., Wanajo, S., Aoki, W., \& Ryan, S. G. 2004, ApJ, 600, L47

Ishimaru, Y., \& Wanajo, S. 1999, ApJ, 511, 33

Johnson, J. A. 2002, ApJS, 139, 219

Kastberg, A., Villemoes, P., Arnesen, A., Heijkenskjœld, F., \& Langereis, A. 1993, J. Opt. Soc. Am. B., 10, 1330

Kostyk, R. I., \& Orlova, T. V. 1983, Astrometriya Astrofiz., 49, 39

Kroll, S., \& Kock, M. 1987, A\&AS, 67, 225

Kurucz, R. 1993, ATLAS9 Stellar Atmosphere Programs and $2 \mathrm{~km} \mathrm{~s}^{-1}$ grid. Kurucz CD-ROM No. 13. Cambridge, Mass.: Smithsonian Astrophysical Observatory

Kurucz, R. L., \& Furenlid, J. 1979, SAO Special Report 387 Sample Special Atlas for Siruis

Lucke, P. B. 1978, A\&A, 64, 367

Maeder, A. 1992, A\&A, 264, 102

Magain, P. 1987, A\&A, 179, 176

Martin, G. A., Fuhr, J. R., \& Wiese, W. L. 1988, J. Phys. Chem. Ref. Data, 17, Suppl. 3

Mashonkina, L. I., Gehren, T., Travaglio, C., \& Borkova, T. 2003, A\&A, 397, 275

Mathews, G. J., \& Cowan, J. J. 1990, Nature, 345, 491

McWilliam, A., Preston, G., W., Sneden, C., \& Searle, L. 1995, ApJ, 109,2736

Mihalas, D., \& Binney, J. 1981, Galactic Astronomy (San Francisco, CA: W. H. Freeman and Co.), Sect. 6.4

Moity, J. 1983, A\&AS, 52, 37 [renormalised by FMW88]
Moore, C. E., Minnaert, M. G. J., \& Houtgast, J. 1966, The solar spectrum $2935 \AA$ to $8770 \AA$ A National Bureau of Standards Monograph (Washington: US Government Printing Office) (USGPO)

Nissen, P. E., Gustafsson, B., Edvardsson, B., \& Gilmore, G. 1994, A\&A, 285, 440

Nissen, P. E., Primas, F, Asplund, M., \& Lambert, D. L. 2002, A\&A, 390,235

Nomoto, K., et al. 1997, Nucl. Phys., A616, 79c13

Nomoto, K., Thielemann, F. K., \& Yokoi, I. 1984, ApJ, 286, 644

Norris, J. E. 2004, Carnegie Observatory Astrophysics Series, Vol. 4, ed. A. Mc William, \& M. Rauch (Cambridge: Cambridge Univ. in press)

Norris, J. E., Ryan, S. G., \& Beers, T. C. 2001, ApJ, 561, 1034

O'Brian, T. R., Wickliffe, M. E., Lawler, J. E., Whaling, W., \& Brault, J. W. 1991, J. Opt. Soc. Am. B, 8, 1185

Oey, M. S. 2003, MNRAS, 339, 849

Prantzos, N. 2003, A\&A, 404, 211

Recchi, S., Matteucci, F., \& D'Ercole, A. 2001, MNRAS, 322, 800

Ryan, S. G. 1989, AJ, 98, 1693

Ryan, S. G., \& Norris, J. E. 1991a, AJ, 101, 1835

Ryan, S. G., Norris, J. E., \& Bessell, M. S. 1991b, AJ, 102, 303

Ryan, S. G., Norris, J. E., \& Beers, T. C. 1996, ApJ, 471, 254

Ryan, S. G., Norris, J. E., \& Beers, T. C. 1999, ApJ, 523, 654 - RNB

Ryan, S. G., Kajino, T., Beers, T. C., et al. 2001, ApJ, 549, 55

Schuster, W. J., \& Nissen, P. E. 1988, A\&AS, 73, 225

Schuster, W. J., \& Nissen, P. E. 1989, A\&A, 222, 65

Schuster, W. J., Parrao, L., \& Contreras Martinez, M. E. 1993, A\&AS, 97, 951

Schuster, W. J., Nissen, P. E., Parrao, L., Beers, T. C., \& Overgaard, L. P. 1996, A\&AS, 117, 317

Shigeyama, T., \& Tsujimoto, T. 2003, ApJ, 598, L47

Smith, G., \& Raggett, D. St J. 1981, J. Phys. B: At. Mol. Phys., 14, 4015

Smith, G., \& O’Neill, J. A. 1975, A\&A, 38, 1

Spite, M., \& Spite, F. 1985, ARA\&A, 23, 225

Thévenin, F., \& Idiart, T. 1999, ApJ, 521, 753

Thielemann, F. K., Hashimoto, M., \& Nomoto, K. 1990, ApJ, 349, 222

Thielemann, F. K., Nomoto, K., \& Hashimoto, M. 1996, ApJ, 460, 408

Tinsley, B. M. 1980, Fundam. Cosmic. Phys., 5, 287

Tozzi, G. P., Brunner, A. J., \& Huber, M. C. E. 1985, MNRAS, 217, 423

Travaglio, C., Gallino, R., Arnone, E., et al. 2004, ApJ, 601, 864

Travaglio, C., Burket, A., \& Galli, D. 2001, ApJS, 277, 211

Tsujimoto, T., \& Shigeyama, T. 1998, ApJ, 508, L151

Tsujimoto, T., Shigeyama, T., \& Yoshii, Y. 1999, ApJ, 519, L63

Unsöld, A., \& Weidemann, V. 1955, Vistas in Astronomy, 1, 1, 249

Wiese, W. L., \& Martin, G. A. 1980, Wavelengths and Transition Probabilities for Atoms and Atomic Ions, NSRDS-NBS No. 68, Part II. (Washington: NBS)

Woosley, S. E., \& Weaver, T. A. 1995, ApJS, 101, 181

Zhao, G., Butler, K., \& Gehren, T. 1998, A\&A, 333, 219 


\section{Online Material}




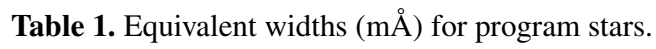

\begin{tabular}{|c|c|c|c|c|c|c|c|c|c|c|c|c|c|}
\hline el & $\bar{\lambda}$ & $\overline{l \log (g f)}$ & ref & $\overline{\mathrm{BD}-13^{\circ} 3442}$ & $\overline{\mathrm{BD}+1^{\circ} 2341 \mathrm{p}}$ & $\overline{\mathrm{BD}+20^{\circ} 2030}$ & $\overline{\mathrm{BD}+24^{\circ} 1676}$ & $\overline{\mathrm{BD}+26^{\circ} 2621}$ & $\overline{\mathrm{BD}+26^{\circ} 3578}$ & $\overline{\mathrm{BD}+3^{\circ} 740}$ & $\overline{\mathrm{BD}+9^{\circ} 2190}$ & CD-24우17504 & $\overline{C D}-33^{\circ} 1173$ \\
\hline $\mathrm{Mg} \mathrm{I}$ & 5172.70 & -0.39 & 7 & 113 & 109 & 118 & 134 & 119 & 140 & 107 & 95 & 79 & 92 \\
\hline Mg I & 5183.62 & -0.17 & 7 & 128 & 122 & 155 & 148 & 134 & 163 & 127 & 119 & 92 & 111 \\
\hline Mg I & 5528.40 & -0.50 & 7 & 20 & 20 & 26 & 32 & 18 & & 21 & 14 & 10 & 10 \\
\hline Mg I & 5711.10 & -1.67 & 8 & & & & & & & & & & \\
\hline $\mathrm{CaI}$ & 5581.98 & -0.56 & 12 & & & & & & & & & & \\
\hline $\mathrm{CaI}$ & 5588.76 & 0.36 & 12 & 20 & 17 & 24 & 24 & & 33 & & 17 & & \\
\hline $\mathrm{CaI}$ & 5590.13 & -0.57 & 12 & & & & & & & & & & \\
\hline $\mathrm{CaI}$ & 6122.22 & -0.31 & 13 & 16 & 19 & 21 & 25 & & & 15 & 12 & & \\
\hline $\mathrm{CaI}$ & 6162.18 & -0.09 & 13 & 21 & 22 & 35 & 35 & 16 & 42 & 21 & 15 & & 13 \\
\hline $\mathrm{CaI}$ & 6169.56 & -0.48 & 12 & & & & & & & & & & \\
\hline $\mathrm{CaI}$ & 6717.69 & -0.52 & 12 & & & & & & & & & & \\
\hline Ti I & 4981.74 & 0.56 & 4,3 & & 12 & 16 & 20 & & 19 & & & & \\
\hline Ti I & 5192.98 & -0.95 & 4 & & & & & & & & & & \\
\hline Ti II & 5185.91 & -1.35 & 10 & & & & 7 & & & & & & \\
\hline CrI & 5206.04 & 0.02 & 10 & & 17 & 25 & 21 & & 27 & & & & 14 \\
\hline $\mathrm{CrI}$ & 5208.43 & 0.16 & 10 & & 21 & 35 & 34 & & 36 & & & & \\
\hline $\mathrm{CrI}$ & 5409.80 & -0.72 & 10 & & & & & & & & & & \\
\hline Fe I & 4920.51 & 0.07 & 11 & & & & & & & 31 & 32 & & \\
\hline $\mathrm{Fe} \mathrm{I}$ & 4938.82 & -1.08 & 11 & & & & & & 8 & & & & \\
\hline $\mathrm{Fe} \mathrm{I}$ & 4966.10 & -0.87 & 11 & & & & & & & & & & \\
\hline $\mathrm{Fe} \mathrm{I}$ & 5041.76 & -2.20 & 11 & & & & & & & & & & \\
\hline $\mathrm{Fe} \mathrm{I}$ & 5049.83 & -1.34 & 11,1 & & & & & & 15 & & & & \\
\hline Fe I & 5068.77 & -1.04 & 11 & & & & 7 & & & & & & \\
\hline $\mathrm{Fe} \mathrm{I}$ & 5083.35 & -2.87 & $11,2,7$ & & & & & & 10 & & & & \\
\hline $\mathrm{Fe} I$ & 5166.28 & -4.20 & 5 & & & & & & & & & & \\
\hline $\mathrm{Fe} \mathrm{I}$ & 5171.61 & -1.76 & 11,6 & 11 & 14 & 16 & 28 & 15 & 26 & 12 & 16 & & 8 \\
\hline $\mathrm{Fe} \mathrm{I}$ & 5191.47 & -0.55 & 11 & & & 12 & 12 & & & & & & \\
\hline Fe I & 5192.35 & -0.42 & 11 & & 9 & 15 & & & 21 & & & & \\
\hline Fe I & 5194.95 & -2.06 & 11,6 & & & 8 & 10 & & 14 & & & & \\
\hline $\mathrm{Fe} I$ & 5198.72 & -2.11 & 11,5 & & & & & & & & & & \\
\hline $\mathrm{Fe} I$ & 5269.55 & -1.33 & 11 & 57 & 54 & 66 & 73 & 61 & & 60 & 54 & 36 & 42 \\
\hline $\mathrm{Fe} \mathrm{I}$ & 5281.80 & -0.83 & 11 & & & & & & 11 & & & & \\
\hline $\mathrm{Fe} \mathrm{I}$ & 5283.63 & -0.48 & 11,2 & 7 & & 11 & 10 & & 14 & & 7 & & \\
\hline $\mathrm{Fe} \mathrm{I}$ & 5302.31 & -0.72 & 2 & & & & & & & & & & \\
\hline $\mathrm{Fe} \mathrm{I}$ & 5324.19 & -0.10 & 2 & & 13 & 20 & 24 & & 27 & 15 & & & \\
\hline $\mathrm{Fe} \mathrm{I}$ & 5328.05 & -1.47 & 11,7 & 48 & 44 & 58 & 68 & & 70 & 48 & 43 & & 38 \\
\hline $\mathrm{Fe} \mathrm{I}$ & 5328.54 & -1.85 & 11 & & 10 & 16 & 15 & & 23 & & & & \\
\hline $\mathrm{Fe} \mathrm{I}$ & 5397.13 & -1.95 & 5 & 22 & 20 & 34 & 37 & 24 & & 25 & 23 & 11 & 14 \\
\hline $\mathrm{Fe} \mathrm{I}$ & 5405.79 & -1.86 & $11,2,7$ & 27 & 22 & 34 & 40 & 28 & & 25 & 23 & 9 & 15 \\
\hline $\mathrm{Fe} \mathrm{I}$ & 5410.92 & 0.40 & 11 & & & & & & 8 & & & & \\
\hline $\mathrm{Fe} I$ & 5415.21 & 0.64 & 11 & 9 & & 10 & 13 & & 16 & & & & \\
\hline $\mathrm{Fe} \mathrm{I}$ & 5429.71 & -1.88 & 11 & 28 & 20 & 35 & 44 & 32 & 46 & 28 & 22 & 11 & 18 \\
\hline $\mathrm{Fe} \mathrm{I}$ & 5434.53 & -2.12 & $11,2,7$ & 17 & 13 & 24 & 25 & 17 & 30 & 16 & 13 & & 10 \\
\hline $\mathrm{Fe} I$ & 5446.92 & -1.88 & 11 & & 18 & 34 & 38 & 28 & 41 & 23 & 24 & 13 & 11 \\
\hline $\mathrm{Fe} I$ & 5569.63 & -0.49 & 1 & & & & & & 10 & & & & \\
\hline $\mathrm{Fe} \mathrm{I}$ & 5572.85 & -0.28 & 2 & & & 9 & & & 16 & & & & \\
\hline $\mathrm{Fe} \mathrm{I}$ & 6136.62 & -1.41 & 11 & & & & & & 11 & & & & \\
\hline $\mathrm{Fe} \mathrm{I}$ & 6191.57 & -1.51 & 11 & & & & & & 9 & & & & \\
\hline $\mathrm{Fe} \mathrm{I}$ & 6678.00 & -1.42 & 11 & & & & & & 7 & & & & \\
\hline Fe II & 4923.93 & -1.32 & 9 & & 38 & 46 & & & & 36 & 36 & & \\
\hline Fe II & 5197.58 & -2.10 & 9 & & & & & & 15 & & & & \\
\hline Fe II & 5276.00 & -1.95 & 9 & & & & 15 & & & 10 & 10 & & \\
\hline
\end{tabular}


Table 1. continued.

\begin{tabular}{|c|c|c|c|c|c|c|c|c|c|c|c|c|c|c|}
\hline$\overline{\mathrm{el}}$ & $\bar{\lambda}$ & CD-35 14849 & ${\mathrm{CD}-71^{\circ} 1234}$ & $\begin{array}{l}\text { CD22943-045 } \\
\end{array}$ & $\begin{array}{c}\text { G126-52 } \\
\end{array}$ & $\begin{array}{l}\text { G4-37 } \\
\end{array}$ & $\begin{array}{l}\text { G64-12 } \\
\end{array}$ & $\begin{array}{l}\text { G64-37 } \\
\end{array}$ & 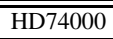 & $\begin{array}{c}\text { HD84937 } \\
\end{array}$ & $\begin{array}{l}\text { LP635-14 } \\
\end{array}$ & $\overline{\mathrm{L} \text { LP651-4 }}$ & LP815-43 & 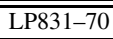 \\
\hline Mg I & 5172.70 & 154 & 135 & 151 & 146 & 140 & 77 & 77 & 192 & 160 & 132 & 125 & 95 & 113 \\
\hline $\mathrm{Mg} \mathrm{I}$ & 5183.62 & 179 & 152 & 177 & 157 & 166 & 92 & 93 & 234 & 180 & 154 & 146 & 118 & 138 \\
\hline $\mathrm{Mg} \mathrm{I}$ & 5528.40 & & 30 & 45 & & & 8 & 10 & & 47 & 33 & 26 & & 21 \\
\hline $\mathrm{Mg} \mathrm{I}$ & 5711.10 & & & & & & & & 9 & & & & & \\
\hline $\mathrm{CaI}$ & 5581.98 & & & 10 & & & & & & & & & & \\
\hline $\mathrm{CaI}$ & 5588.76 & & 25 & 36 & 32 & 22 & & & 41 & 36 & 24 & 14 & & \\
\hline $\mathrm{CaI}$ & 5590.13 & & & 8 & & & & & 11 & & & & & \\
\hline $\mathrm{CaI}$ & 6122.22 & & 21 & & & & & & & 35 & 19 & & & \\
\hline $\mathrm{CaI}$ & 6162.18 & 36 & 34 & 43 & 38 & & 10 & 9 & 57 & 46 & 31 & 23 & 17 & \\
\hline $\mathrm{CaI}$ & 6169.56 & & & 10 & & & & & 13 & & & & & \\
\hline $\mathrm{CaI}$ & 6717.69 & & & & & & & & & 8 & & & & \\
\hline Ti I & 4981.74 & & & 20 & 18 & 14 & & & 29 & 23 & & & & 11 \\
\hline Ti I & 5192.98 & & & & & & & & 8 & & & & & \\
\hline Ti II & 5185.91 & & & 8 & & & & & & 10 & & & & \\
\hline CrI & 5206.04 & 27 & 22 & 29 & 31 & 27 & & & 45 & 35 & 22 & & & \\
\hline $\mathrm{CrI}$ & 5208.43 & & 34 & 40 & & 30 & & & 65 & & 32 & & & 15 \\
\hline CrI & 5409.80 & 7 & 14 & & & & & & 14 & 9 & & & & \\
\hline $\mathrm{Fe} \mathrm{I}$ & 4920.51 & & & & & & & & & & & & & \\
\hline $\mathrm{Fe} \mathrm{I}$ & 4938.82 & 8 & 7 & 7 & 8 & & & & 19 & 8 & & & & \\
\hline Fe I & 4966.10 & & & & & & & & 15 & & & & & \\
\hline Fe I & 5041.76 & & 11 & & & & & & & & & & & \\
\hline $\mathrm{Fe} \mathrm{I}$ & 5049.83 & 15 & 12 & 14 & 16 & 13 & & & 30 & 19 & 15 & 9 & & \\
\hline Fe I & 5068.77 & 9 & & & 8 & & & & 18 & & & & & \\
\hline Fe I & 5083.35 & 11 & & & 10 & & & & 17 & 9 & 8 & & & \\
\hline $\mathrm{Fe} \mathrm{I}$ & 5166.28 & & & & & & & & 8 & & & & & \\
\hline $\mathrm{Fe} \mathrm{I}$ & 5171.61 & 29 & 23 & 25 & & 23 & & 8 & 42 & 32 & 20 & 14 & 11 & 11 \\
\hline $\mathrm{Fe} \mathrm{I}$ & 5191.47 & 14 & 15 & 17 & 18 & & & & 31 & 22 & 9 & & & 9 \\
\hline $\mathrm{Fe} \mathrm{I}$ & 5192.35 & 21 & 18 & 19 & 24 & 14 & & & 39 & & 15 & & & \\
\hline $\mathrm{Fe} \mathrm{I}$ & 5194.95 & 16 & 13 & 14 & 14 & & & & 25 & 19 & 10 & 14 & & \\
\hline $\mathrm{Fe} \mathrm{I}$ & 5198.72 & & & & & & & & 8 & & & & & \\
\hline $\mathrm{Fe} \mathrm{I}$ & 5269.55 & & 73 & & & & 32 & 34 & & & 70 & 56 & & 52 \\
\hline $\mathrm{Fe} \mathrm{I}$ & 5281.80 & 10 & & 13 & & 8 & & & 20 & 11 & 7 & & & \\
\hline $\mathrm{Fe} \mathrm{I}$ & 5283.63 & 15 & 11 & 17 & 15 & 9 & & & 29 & 18 & 12 & 9 & & \\
\hline $\mathrm{Fe} \mathrm{I}$ & 5302.31 & 10 & & 8 & 9 & & & & 16 & 8 & & & & \\
\hline $\mathrm{Fe} \mathrm{I}$ & 5324.19 & 26 & 22 & 27 & 25 & 22 & & & 42 & 32 & 20 & 15 & & \\
\hline $\mathrm{Fe} \mathrm{I}$ & 5328.05 & & 62 & 68 & 71 & 67 & & 31 & 88 & 77 & 64 & 47 & 38 & 41 \\
\hline $\mathrm{Fe} \mathrm{I}$ & 5328.54 & & 21 & 23 & 25 & 16 & & & 41 & 29 & 17 & 8 & & \\
\hline $\mathrm{Fe} \mathrm{I}$ & 5397.13 & & 37 & 43 & & & 9 & 12 & & & 39 & 27 & & 23 \\
\hline $\mathrm{Fe} \mathrm{I}$ & 5405.79 & 49 & 42 & 47 & & 38 & 13 & 15 & 68 & 52 & 38 & 28 & 21 & 19 \\
\hline $\mathrm{Fe} \mathrm{I}$ & 5410.92 & & 10 & 8 & 8 & & & & 16 & 10 & & & & \\
\hline $\mathrm{Fe} \mathrm{I}$ & 5415.21 & 17 & 13 & 15 & 16 & & & & 29 & 19 & 12 & & & \\
\hline $\mathrm{Fe} \mathrm{I}$ & 5429.71 & 48 & 44 & 46 & 48 & 39 & 9 & 13 & 69 & 55 & 39 & 31 & 21 & 24 \\
\hline $\mathrm{Fe} \mathrm{I}$ & 5434.53 & 33 & 27 & 31 & 35 & 25 & & 8 & 51 & 39 & 26 & 18 & 13 & 14 \\
\hline $\mathrm{Fe} \mathrm{I}$ & 5446.92 & & 40 & 44 & 47 & 37 & & 13 & 65 & 51 & 37 & 27 & 21 & 21 \\
\hline $\mathrm{Fe} \mathrm{I}$ & 5569.63 & 10 & 9 & 9 & 9 & & & & 20 & 15 & 8 & & & \\
\hline $\mathrm{Fe} \mathrm{I}$ & 5572.85 & 16 & 11 & 16 & 14 & & & & 31 & 23 & 11 & & 7 & \\
\hline Fe I & 6136.62 & 13 & 8 & & 10 & & & & 25 & 16 & 10 & 9 & & \\
\hline $\mathrm{Fe} \mathrm{I}$ & 6191.57 & & & & & & & & 20 & 13 & 9 & & & \\
\hline Fe I & 6677.80 & 8 & & 11 & & & & & 17 & 11 & 7 & & & \\
\hline Fe II & 4923.93 & & 48 & & & & & 22 & & 63 & & & & \\
\hline Fe II & 5197.58 & 9 & 8 & 14 & 12 & & & & 15 & 15 & & & & \\
\hline Fe II & 5276.00 & 12 & 12 & 15 & & & & & & 20 & 10 & & & \\
\hline
\end{tabular}

